\title{
Model design and parameter optimization of CNN for side- channel cryptanalysis
}

\author{
Yun Lin Liu ${ }^{\text {Corresp., }}{ }^{1}$, Yan Kai Chen ${ }^{1}$, Wei Xiong Li ${ }^{1}$, Yang Zhang ${ }^{1}$ \\ ${ }^{1}$ Center of Equipment Simulation Training, Shijiazhuang campus of the Army Engineering University, Shijiazhuang, Hebei, China \\ Corresponding Author: Yun Lin Liu \\ Email address: Ilyun324@163.com
}

Background. The side-channel cryptanalysis method based on convolutional neural network (CNNSCA) can effectively carry out cryptographic attacks. The CNNSCA network models that achieve cryptanalysis mainly include CNNSCA based on the VGG variant (VGG-CNNSCA) and CNNSCA based on the Alexnet variant (Alex-CNNSCA). The learning ability and cryptanalysis performance of these CNNSCA models are not optimal, and the trained model has low accuracy, too long training time, and takes up more computing resources. In order to improve the overall performance of CNNSCA, the paper will improve CNNSCA model design and hyperparameter optimization.

Methods. The paper first studied the CNN architecture composition in the SCA application scenario, and derives the calculation process of the CNN core algorithm for side-channel leakage of one-dimensional data. Secondly, a new basic model of CNNSCA was designed by comprehensively using the advantages of VGG-CNNSCA model classification and fitting efficiency and Alex-CNNSCA model occupying less computing resources, in order to better reduce the gradient dispersion problem of error back propagation in deep networks, the SE (Squeeze-and-Excitation) module is newly embedded in this basic model, this module is used for the first time in the CNNSCA model, which forms a new idea for the design of the CNNSCA model.Then apply this basic model to a known first-order masked dataset from the side-channel leak public database (ASCAD). In this application scenario, according to the model design rules and actual experimental results, exclude non-essential experimental parameters. Optimize the various hyperparameters of the basic model in the most objective experimental parameter interval to improve its cryptanalysis performance, which results in a hyper-parameter optimization scheme and a final benchmark for the determination of hyper-parameters.

Results. Finally, a new CNNSCA model optimized architecture for attacking unprotected encryption devices is obtained--CNNSCAnew. Through comparative experiments, CNNSCAnew's guessing entropy evaluation results converged to 61 . From model training to successful recovery of the key, the total time spent was shortened to about 30 minutes, and we obtained better performance than other CNNSCA models. 
1 Model design and parameter optimization of CNN for 2 side-channel cryptanalysis

3

4

Yun Lin Liu, Yan Kai Chen, Wei Xiong Li, Yang Zhang

email address of the corresponding author(llyun324@163.com)

Center of Equipment Simulation Training,Shijiazhuang campus of the Army Engineering University, Shijiazhuang, 050003, China

\section{Abstract}

Background. The side-channel cryptanalysis method based on convolutional neural network (CNNSCA) can effectively carry out cryptographic attacks. The CNNSCA network models that achieve cryptanalysis mainly include CNNSCA based on the VGG variant (VGG-CNNSCA) and CNNSCA based on the Alexnet variant (Alex-CNNSCA). The learning ability and cryptanalysis performance of these CNNSCA models are not optimal, and the trained model has low accuracy, too long training time, and takes up more computing resources. In order to improve the overall performance of CNNSCA, the paper will improve CNNSCA model design and hyperparameter optimization.

Methods. The paper first studied the CNN architecture composition in the SCA application scenario, and derives the calculation process of the CNN core algorithm for side-channel leakage of one-dimensional data. Secondly, a new basic model of CNNSCA was designed by comprehensively using the advantages of VGG-CNNSCA model classification and fitting efficiency and Alex-CNNSCA model occupying less computing resources, in order to better reduce the gradient dispersion problem of error back propagation in deep networks, the SE (Squeeze-and-Excitation) module is newly embedded in this basic model, this module is used for the first time in the CNNSCA model, which forms a new idea for the design of the CNNSCA model.Then apply this basic model to a known first-order masked dataset from the side-channel leak public database (ASCAD). In this application scenario, according to the model design rules and actual experimental results, exclude non-essential experimental parameters. Optimize the various hyperparameters of the basic model in the most objective experimental parameter interval to improve its cryptanalysis performance, which results in a hyper-parameter optimization scheme and a final benchmark for the determination of hyper-parameters. Results. Finally, a new CNNSCA model optimized architecture for attacking unprotected encryption devices is obtained C CNNSCAnew. Through comparative experiments, CNNSCAnew's guessing entropy evaluation results converged to 61 . From model training to successful recovery of the key, the total time spent was shortened to about 30 minutes, and we obtained better performance than other CNNSCA models.

Key words Side-channel analysis,CNN,VGG, Alexnet,SEnet, Hyperparameter 


\section{Introduction}

41 Side Channel Analysis ${ }^{[1]}(\mathrm{SCA})$ refers to bypassing the tedious analysis of encryption algorithms,

42

43

44

45

46

47

48

49

50

51

52

53

54

55

56

57

58

59

60

61

62

63

64

65

66

67

68

69

70

71

72

73

74

75

76

77

78

79 by using the information (such as execution time, power consumption, electromagnetic radiation , etc.)leaked by the hardware device embedded in the encryption algorithm during the calculation process , combined with statistical analysis methods to attack cryptographic systems. The sidechannel cryptanalysis method is divided into profiling methods and non-profiling methods: nonprofiling methods include differential power $\operatorname{attack}^{[2]}$ (DPA), correlation power attack ${ }^{[3]}(\mathrm{CPA})$ and mutual information attack ${ }^{[4]}$ (MIA); profiling methods include template $\operatorname{attack}^{[5]}$ (TA), sidechannel cryptography attack based on multi-layer perceptron (MLPSCA), and side-channel cryptography attack based on convolutional neural networks (CNNSCA). Although the attack method of the non-profiling method is simple and direct, weak side-channel signal or excessive environmental noise can cause the attack to fail. The profiling method can effectively analyze the characteristics of the side-channel signal when the encryption knowledge of the attacking device is obtained in advance, so it is easier to crack the cryptogramme. In the case of an encrypted implementation copy, the best cryptanalysis attack in the traditional SCA method is TA ${ }^{[5-8]}$, but TA has difficulties in statistical analysis when processing high-dimensional side-channel signals, and cannot attack the implementation of protected encryption. With the rapid development of supervised machine learning algorithms, it can effectively analyze one-dimensional data with similar power consumption traces in other fields, and side-channel cryptanalysis based on machine learning (MLSCA $)^{[9-11]}$ has begun to emerge. The new profiling method MLPSCA surpasses the traditional profiling method in attack performance ${ }^{[11-13]}$, and overcomes the shortcomings of template attacks that cannot handle high-dimensional side-channel signals, but it also loses effectiveness when attacking encryption with protection. Nowadays, with the development of machine learning, deep learning techniques with excellent performance in image classification and target recognition have become popular. Studies have shown that the application of convolutional neural network algorithms under deep learning can produce better encryption performance in side-channel analysis ${ }^{[12-16]}$. The deep network helps to mine the deep features in the data, which can make the neural network have more powerful performance, which makes CNNSCA can also attack the encryption implementation with protection. In the sidechannel analysis application scenario, deep learning eliminates the step of manually extracting features from the workflow of model construction. For example, in the traditional bypass attack method, the TA with better attack effect only selects 5 strong feature points, while the deep learning model can select hundreds to thousands of feature points, select more features to construct a template, it is extremely beneficial to the generalization and robustness of the sidechannel analysis model.

Analyze the above domestic and foreign documents, there are two main types of CNN structures that have successfully used CNNSCA to achieve cryptanalysis, which are based on two variants of Alexnet and VGGnet network structures [12,16-18]. Among them, the 2012 ILSVRC(ImageNet Large Scale Visual Recognition Challenge) champion structure Alexnet ${ }^{[19]}$, although successful 
80 in the SCA application, but in fact, the training accuracy of CNNSCA based on this network

81 variant is not high, moreover, the Alex-CNNSCA network model in the literature [16] has a large

82 amount of training parameters and a long calculation time, which means that there is still room

83 for optimization of this network structure. The 2013 ILSVRC champion network ZFNet ${ }^{[20]}$ has

84 not changed much from the 2012 first ILSVRC champion network Alexnet. The 2014 ILSVRC

85 runner-up structure VGGnet ${ }^{[21]}$ also succeeded in breaking secrets in the SCA application. In the

86 literature [12,17-18], VGG-CNNSCA models with different parameters were proposed. Among

87 them, the best cryptanalysis performance is in the literature [12] proposed VGG-CNNSCA, but

88 its training accuracy is still not high. Obviously, there is still room for improvement in the

89 cryptanalysis performance. The 2014 ILSVRC champion network GoogLeNet ${ }^{[22]}$ and the 2015

90 ILSVRC champion network ResNet ${ }^{[23]}$ have also been used in SCA, but the effect is average.

91 This conclusion has been confirmed in the literature [12]. The last ILSVRC champion network in

922017 was the SEnet ${ }^{[24]}$ proposed by Momenta and Oxford University. There is currently little

93 literature on applying this network to SCA scenarios.

94

95

96

97

98

99

100

101

Although CNNSCA overcomes the shortcomings of the previous profiling methods and improves the cryptanalysis performance, the existing CNNSCA model learning ability and cryptanalysis performance are not optimal. The disadvantages of these models are: low training accuracy and excessive training time long, taking up too much computing resources, etc. The reason is mainly affected by CNNSCA model design and hyperparameter optimization. In order to improve the overall performance of CNNSCA, the paper will improve CNNSCA model design and hyperparameter optimization, and has done the following work:

102

1. The composition of the CNN architecture in the SCA application scenario is studied, and the calculation process of the $\mathrm{CNN}$ core algorithm for side-channel leakage of onedimensional data is deduced.

2. Taking advantage of the high efficiency of classification and fitting of the VGGCNNSCA model and the advantages of the Alex-CNNSCA model occupying less computing resources, a new basic model of CNNSCA is designed to better reduce the gradient dispersion of error back propagation in the deep network. The problem is that the SE module is newly embedded in this basic model, so that the model basically achieves the purpose of breaking the secrets, thereby solving the problem of constructing the CNNSCA model.

3. Apply the above basic model to a known first-order mask data set of the side-channel leak public database (ASCAD). In this application scenario, according to the model design rules and actual experimental results, unnecessary experiments are maximized parameter, optimize the various hyperparameters of the model in the most objective experimental parameter interval to improve the breaking performance of the new CNNSCA, which solves the problem of hyperparameter optimization, and gives the final determination benchmark for hyperparameters. Finally, a new CNNSCA model 
119

120

121

122

123

124

125

126

127

128

129

130

131

132

133

134

135

136

137

138

139

140

141

142

143

144

145

146

147

148

149

150

151

152

153

154

155

156

157

158 optimized architecture for attacking unprotected encryption devices-CNNSCAnew is obtained.

4. The performance verified on public data sets exceeds other profiling SCA methods.

The algorithms involved in the paper experiments are all programmed in the Python language, and use the deep learning architecture Keras library ${ }^{[25]}$ (version 2.4.3) or directly use the GPU version of the Tensorflow library ${ }^{[26]}$ (version 2.2.0). The experiment was carried out on an ordinary computer equipped with 16 GB RAM and 8G GPU (Nvidia GF RTX 2060). All experiments use side-channel leaking public data sets-known first-order mask data sets in the ASCAD database, use 50,000 pieces of data from its training set to train the model, and randomly select 1,000 pieces of data from its test set for testing. When testing the cryptanalysis performance of the CNNSCA model, the guessing entropy index is used to evaluate the cryptanalysis performance.

\section{Materials \& Methods}

\section{Materials}

\section{$1 \mathrm{CNN}$}

Convolutional Neural Network (CNN) is one of the most successful algorithms of artificial intelligence, and it is a multi-layer neural network with a new structure. Its design is inspired by the research on the optic nerve receptive field ${ }^{[27-28]}$. The core component of CNN, the convolution kernel, is the structural embodiment of the local receptive field. It belongs to the deep network of back propagation training. It uses the two-dimensional spatial relationship of the data to reduce the number of parameters that need to be learned, and improves the training performance of the BP algorithm(Error Back Propagation, which is used to calculate the gradient of the loss function with respect to the parameters of the neural network) to a certain extent. The main difference between CNN and MLP is the addition of the convolution block structure. In the convolution block, a small part of the input data is used as the original input of the network structure, and the data information is forwarded layer by layer in the network, and each layer uses several convolution cores to extract features of the input data. Convolutional neural networks have been successfully applied in computer vision, natural language processing, disaster climate prediction and other fields, especially shine on ILSVRC ${ }^{[29]}$. ILSVRC is one of the most popular and authoritative academic competitions in the field of machine vision in recent years, representing the highest level in the field of imaging. The introduction of outstanding CNNs in the image classification and target positioning projects of the ILSVRC competition over the years is shown in Table $1(\mathrm{CNN}$ with outstanding performance in previous ILSVRC competitions).

Table 1 sorts out the champion networks and individual runner-up networks of the last ILSVRC classification task from 2012 to 2017, and briefly introduces their names, rankings, classification results under the top1 and top5 indicators, and some remarks. Top1 refers to the largest 
159

160

161

162

163

164

165

166

167

168

169

170

171

172

173

174

175

176

177

178

179

180

181

182

183

184

185

186

187

188

189

190

191

192

193

194

195

196

197

probability vector as the prediction result, if the classification is correct, it is correct. Top5 is correct as long as there is a correct classification in the top five of the largest probability vectors. Among them, the error rate of the classification results of the last champion network SEnet (2017) under the top5 index is obviously the lowest, reaching $2.25 \%$. Deep convolutional networks have greatly promoted the development of various fields of deep learning.

\section{CNNSCA model hyperparameters}

Hyperparameters of neural network models are a concept often used in machine learning or deep learning, including the structural parameters and training parameters of the network model. To design a CNN model in SCA application scenarios, all the parameters that need to be set are as follows:

1) Structural parameters

Define all the parameters of the neural network architecture, including the regular parameter network layer activation function, classification function, loss function, and optimizer. In the convolutional neural network, the network layer is subdivided into convolutional blocks (a combination of different numbers of convolutional layers and pooling layers), convolutional layers, full link layers, pooling layers, the number of convolution kernels, convolution kernel size and fill.

The convolution block, convolution layer, pooling layer, number of convolution kernels, convolution kernel size and padding in these parameters mainly control the scale and performance of feature extraction in the feature extraction stage of the CNNSCA model. Full link layer, activation function, classification function and loss function, these parameters constitute the main body of the CNNSCA network, and perform feature learning and fitting classification on side-channel leakage data.

\section{2) Training parameters}

Control the parameters of the network model training phase, including the number of iterations, batch learning volume, and learning rate. When training a network model, a complete training set is processed at one time, which is called complete batch learning. If a single training sample is processed at a time, it is called random learning. In practice, in order to improve efficiency, a compromise method is usually adopted, called small-batch learning, that is, small batches of training samples are processed at one time during the model learning process. The batch size depends on environmental factors ${ }^{[30]}$ (such as network architecture, computer GPU performance, the trade-off between network regularization effect and stability, etc.). The number of iterations is an important parameter to be adjusted. A small value will cause the network model to underfit (the model is too poor to capture the feature trend in the training data set), while a higher value will cause the network model to overfit (the model is too Complex, perfectly fits the training data set, but cannot generalize its prediction to other data sets). In addition, the variable that optimizes 
198

199

200

201

202

203

204

205

206

207

208

209

210

211

212

213

214

215

216

217

218

219

220

221

222

223

224

225

226

227

228

229

230

231

232

233

234

235

236

237

the training effect of the network model-the learning rate (also called the step size), aims to promote the gradient (ie, the error gradient) drop during the training process.

The number of iterations and the amount of batch learning affect the degree of model training, and the optimizer and learning rate are used to control the gradient of the error. These parameters all have an important impact on CNNSCA's cryptanalysis performance and need to be adjusted according to specific attack scenarios.

\section{Core algorithm and network structure of CNNSCA}

\subsection{CNN network structure for SCA}

Combined with the side-channel cryptanalysis scenario, the CNN applied to the side-channel attack mainly has six network layers stacked layer by layer and an embeddable SE module:

a) Convolutional layers (Conv for short) are linear layers. The incomplete connection between layers can avoid the two shortcomings of a fully connected network: training weights requires a huge amount of calculation and model overfitting. The weights of the same convolution kernel (also known as filters) in the same layer are shared, allowing the convolution layer to extract constant displacement features while reducing parameters. The convolutional layer can also use multiple convolution kernels. Each convolution kernel extracts different abstract features from the input vector. These abstract features are arranged side by side in an additional dimension (the so-called depth), making the $\mathrm{CNN}$ resistant to time-domain distortion Vector features $^{[31]}$. The convolutional layer usually needs to set the padding mode, one is valid padding, so that the dimension of the feature vector after convolution is smaller than the original vector; the other is the same padding, so that the convolutional The feature vector dimension is the same as the original vector.

b) Batch Normalization layers ${ }^{[32]}$ (BN for short), whose role is to reduce the deviation of covariates in the two stages of training and prediction, which is conducive to the use of a higher learning rate for the network model ${ }^{[33]}$.

c) Activation layers (ACT for short) are non-linear layers and consist of a single real function, which acts on each coordinate of the input vector. The ReLU function is currently the first choice in deep learning.

d) Pooling layers (POOL for short) are non-linear layers. Use the pooling window to slide on the input vector to extract salient feature points to reduce the feature dimension. There is no weight in the pooling layer, which will not cause distortion of the input signal.

e) Fully-Connected layers (FC for short), the neurons between the layers are completely connected, and these layers need to train a lot of weights. This layer is expressed by an affine function as: D-dimensional $\mathrm{x}$ vector is the input, and $\mathrm{Ax}+\mathrm{B}$ is the output. Among them, $A \in R C \times D$ is the weight matrix and $B \in R C$ is the deviation vector. These weights and deviations are the training parameters of the FC layer. 
238

239

240

241

242

243

244

245

246

247

248

249

250

251

252

253

254

255

256

257

258

259

260

261

262

263

264

265

266

267

268

269

270

271

272

273

274

275

276

277

f) Softmax layer (SOFT for short). In multi-classification tasks, softmax is usually used as the activation function of the output layer. Here, softmax is used to represent the output layer. This layer classifies the input, obtains the predicted value of each label, and takes the label corresponding to the maximum value as the global classification result.

g) SE module, SEnet is a classic attention model structure, and it is also a required basic network structure for fine-grained classification tasks. SEnet proposed the Squeezeand-Excitation (SE) module, which did not introduce a new spatial dimension, and improved the representation ability of the model by displaying the channel correlation between the features of the convolutional layer. The feature recalibration mechanism: by using global information to selectively enhance informatized features and compress those useless features at the same time. In deep network training, this mechanism can effectively overcome the gradient dispersion problem in error back propagation. The SE module is universal. Even if it is embedded in an existing model, its parameters do not increase significantly. It is a relatively successful attention module ${ }^{[24]}$. The structure of the SE module is shown in Figure 1 (SE module).

In Figure 1, the SE module uses global pooling as a squeeze operation, and then uses two FC layers to form an excitation structure to profile the correlation between channels, and output and input the same number of feature channels weights. The advantages of this are: 1) it has more nonlinearity and can better fit the complex correlation between channels; 2) the amount of parameters and the amount of calculation are greatly reduced. Then obtain the normalized weight between 0 and 1 through a sigmoid function, and then use a scale operation to weight the normalized weight to the features of each channel ${ }^{[24]}$. Finally, the output of scale is superimposed on the input $x$ before the SE module to generate a new vector $2 /$

\subsection{Core algorithm of $\mathrm{CNN}$ for SCA}

1) Convolution calculation

Usually convolution operations in the field of computer vision are numerical operations on twodimensional image data. In the SCA application scenario, the dimensionality of the convolution operation is adjusted, which is to slide the convolution kernel on the one-dimensional energy trace data. The number of steps moved each time is called the step length, and the convolution calculation is performed on each sliding to obtain a value. After one round of calculation is completed, a feature vector representing the vector feature is obtained. The rule of numerical operation is to multiply a one-dimensional convolution kernel with a value at the corresponding position of a one-dimensional vector, and then sum. For example, there is a $1 \times 3$ convolution kernel, which convolves a $1 \times 6$ one-dimensional vector with a step size of 1 . The calculation process is shown in Figure 2 (Convolution calculation process).

Peer) Comput. Sci. reviewing PDF | (CS-2021:09:65519:1:1:NEW 20 Nov 2021) 
278

279 In Figure 2(a), the convolution kernel slides from the left side of the input vector. The first

280

281

282

283

284

285

286

287

288

289

290

291

292

293

294

295

296

297

298

299

300

301

302

303

304

305

306

307

308

309

310

311

312

313

314

315 numerical calculation is: $1 \times 1+0 \times 0+1 \times 1=2$, and the first value 2 of the new feature vector is obtained. Then, the convolution kernel slides one step to the right to continue the numerical calculation: $1 \times 0+0 \times 1+1 \times 0=0$, to get the second value 0 of the new feature vector, as shown in Figure 2(b). Repeat this process until the convolution kernel slides to the far right of the input vector, and the convolution calculation is complete.

\section{2) Pooling calculation}

There are three ways of pooling: Max-Pooling, Mean-Pooling and Stochastic Pooling. Maximum pooling is to extract the maximum value of the value in the pooling window, average pooling is to extract the average value of the value in the pooling window, and random pooling is to randomly extract the value in the pooling window. The original pooling operation of CNN is also a numerical operation on two-dimensional image data. In the SCA application scenario, the pooling calculation has also been dimensionally adjusted, and a pooling mode is selected for calculation on the one-dimensional energy trace data. For example, the pooling window size is $1 \times 2$, and the maximum or average pooling operation is performed on a $1 \times 6$ one-dimensional vector with a step size of 2. The pooling calculation is shown in Figure 3 (Pooling calculation process).

In Figure 3(a), the maximum pooling starts from the left side of the input vector. Every two steps of the pooling window, the maximum value of the two values in the window is selected as a value of the new feature vector. The average pooling is shown in Figure 3(b). For every two sliding steps of the pooling window, the average of the two values of the window class is calculated as a value of the new feature vector. The pooling window slides to the right until the rightmost of the input vector, and the pooling calculation is complete.

\section{3) softmax function}

This function normalizes the output value and converts all output values into probabilities. The sum of the probabilities is 1 . The formula of softmax is:

$\operatorname{softmax}\left(x_{i}\right)=\frac{\exp \left(x_{i}\right)}{\Sigma_{j} \exp \left(x_{j}\right)}$

Here $x_{i}$ represents the input of the i-th neuron in the softmax layer, $x_{j}$ represents the input of the $\mathrm{j}$-th neurons in the softmax layer, and $\sum_{j}$ is the sum of calculations for $x_{j}$. The result of the function is used as the fitting probability of the i-th neuron label.

4) Principle of weight adjustment

Using the cost function and gradient descent algorithm ${ }^{[34]}$, each time the network model is trained, the weights are automatically adjusted in the direction of error reduction, so that the 
316 training parameters are repeated until all iterations are over, and the weight adjustment is 317 completed.

318

319 5) Evaluation of Cryptanalysis Performance

320 Generally, security officers consider two indicators when evaluating CNNSCA's cryptanalysis

321

322

323

324

325

326

327

328

329

330

331

332

333

334

335

336

337

338

339

340

341

342

343

344

345

346

347

348

349

350

351

352

353

354

performance: one is the training accuracy of the neural network model during modeling, the Acc indicator $^{[35]}$, and the other is the security indicator guessing entropy of the key obtained in the attack phase ${ }^{[36-37]}$. The guessing entropy index is commonly used to evaluate the SCA cryptanalysis performance, and the guessing entropy is used to measure the efficiency of decrypt.Guessing Entropy (GE) is obtained through a custom rank function $\operatorname{Rank}(\cdot)$, which is defined as:

$\operatorname{Rank}\left(\hat{g}, D_{\text {train }}, D_{\text {test }}, n\right)=\left|\left\{k \in K \mid d_{n}[k] \geq d_{n}\left[k^{*}\right]\right\}\right|$

The adversary uses the modeling data set $D_{\text {train }}$ to establish a bypass analysis model $g$, and uses $n$ energy trace samples in the attack data set $D_{\text {test }}$ to perform $n$ attacks during the attack phase.

After each attack, the logarithm value of the distribution probability of 256 types of hypothetical cryptograms is obtained, compose a vector $d_{i}=\left[d_{i}[1], d_{i}[2], \mathrm{L}, d_{i}[k]\right]$, whose indexes are arranged in the positive order of the hypothetical cryptogramme's key space (the index counts from zero), where $i \in n, k \in K$, and $K$ is the key space of the hypothetical cryptogramme. The results of each attack are accumulated. Then, the rank function $\operatorname{Rank}(\cdot)$ sorts all the elements of the vector $d_{i}$ in reverse order by value, and keeps the position of the corresponding index of each element in the vector before and after sorting consistent with the position of the element, and obtains a new ranking vector $D_{i}=\left[D_{i}[1], D_{i}[2], \mathrm{L}, D_{i}[k]\right]$, where each the element $D_{\mathrm{i}}[k]$ contains two values $k$ and $d[k]$, and finally the index of the logarithmic element of the known cryptogramme $k^{*}$ probability in $D_{i}$ is output, that is, the guessing entropy $\mathrm{GE}\left(d\left[k^{*}\right]\right)$. At the i-th attack, the higher the matching rate of the energy trace model of the real cryptogramme, the higher the index ranking of its $\operatorname{GE}\left(d\left[k^{*}\right]\right)$. Guessing entropy is the $\mathrm{GE}\left(d\left[k^{*}\right]\right)$ index ranking output of each attack- - rank. In $n$ attacks, the better the performance of the cryptanalysis method and the higher the efficiency, the faster the ranking of $\operatorname{GE}\left(d\left[k^{*}\right]\right)$ converge to zero. It shows that in the $\mathrm{i}$-th attack, the guessing entropy converges to zero and continues to converge in subsequent attacks. The adversary only needs $i$ attacks to crack the cryptogramme, that is, only $i$ power consumption traces are needed to break the secret. Equation (2) can be rewritten as (3):

$\mathrm{GE}_{n}(\hat{g})=\operatorname{Every}\left[\operatorname{Rank}\left(\hat{g}, D_{\text {train }}, D_{\text {test }}, n\right)\right]$

4 Side-channel leaking public data sets

The newly published ASCAD database ${ }^{[12]}$ aims to achieve AES-128 with first-order mask protection, namely 8-bit AVR microcontroller (ATmega8515), in which the energy trace is the data signal converted by the collected electromagnetic radiation. The adversary outputs the collected signal for the third S-box of the first round of AES encryption, and launches an attack

Peer] Comput. Sci. reviewing PDF | (CS-2021:09:65519:1:1:NEW 20 Nov 2021) 
355

356

357

358

359

360

361

362

363

364

365

366

367

368

369

370

371

372

373

374

375

376

377

378

379

380

381

382

383

384

385

386

387

388

389

390

391

392

393

394

against the first AES key byte. The database follows the MNIST database ${ }^{[38]}$ rules and provides a total of four data sets, each with 60,000 entries power consumption traces, of which 50,000 power consumption traces are used for analysis/training, and 10,000 power consumption traces are used for testing/attack. The first three ASCAD data sets respectively represent the encryption realization leakage with three different random delay protection countermeasures. The signal offsets desync $=0$, desync $=50$, and desync $=100$ are used to represent these three data sets with two strategies of mask and delay. All power consumption traces in the first three types of data sets contain 700 feature points. These feature points are selected from the original energy trace containing 100,000 feature points, and the selection basis is the position of the largest signal peak. When the mask is known, the maximum signal-to-noise ratio of the data set can reach 0.8 , but it is almost 0 when the mask is unknown. The last ASCAD data set stores the original energy trace.

\section{Methods}

\section{Design of CNNSCA base model}

With reference to the advantages of the VGG-CNNSCA model with high classification and fitting efficiency and the Alex-CNNSCA model occupying less computing resources, the paper selects the same structural parameters from these two models, and some of the factors that promote the high fitting efficiency of the two types of models parameter. These parameters construct a new CNN simple model specifically for SCA scenarios, which is used to test the impact of different hyperparameters on model performance. The convolution block of this simple model consists of the Conv layer, BN layer, and ACT layer. After the block, a POOL layer is usually added to reduce the feature dimension. The new convolution block is repeated $\mathrm{n}$ times in the network model until it is reasonable. Until the output of the size. Then, introduce $n$ FC layers, use the softmax function in the last FC layer, and finally output the classification prediction results. In addition, in order to improve the classification and recognition performance of the CNNSCA model, the SE module is newly embedded in the simple model. Its main function is to reduce the gradient dispersion problem in error back propagation. This is the first use in the CNNSCA model. The SE module will be embedded between the convolutional layer and the pooling layer of the convolutional block of the simple model, and the simple model containing the SE module will be renamed to the CNNSCA base model-CNNSCAbase. The newly designed CNNSCAbase structure is shown in Figure 4(Convolutional network structure in a side-channel attack scenario).

The initial configuration basis and selection of CNNSCAbase are as follows: Find out the two prototypes of Alex-CNNSCA and VGG-CNNSCA to set the same parameters, set these parameters in CNNSCAbase in the same way, these parameters are as follows: 5 convolutional blocks , 3 full connections, the padding modes of the convolutional layers are SAME, and the activation functions of all layers before the last layer of the network select ReLU. In addition, in most classification tasks, convolutional networks use softmax, crossentropy, and RMSprop as the 
395

396

397

398

399

400

401

402

403

404

405

406

407

408

409

410

411

412

413

414

415

416

417

418

419

420

421

422

423

424

425

426

427

428

429

430

431

432

433

model's classification function, loss function, and optimizer ${ }^{[19-23]}$. Here, CNNSCAbase also chooses to use these three activation functions. Since the side-channel leakage data belongs to one-dimensional data, the processing complexity is less than that of two-dimensional data. Here, the convolution layer of each convolution block is initialized to 1 , and the number of convolution kernels in the first convolution layer is 64 (choose the smaller number of Alexnet or VGGnet), the size of the convolution kernel is $3 \times 3$, the step size is 1 , the pooling mode is tentatively averaged pooling mode, the pooling window size is 2, and the step size is 2 . In addition, in the initial setting of CNNSCAbase, a new SE module is embedded in the last four convolution blocks. All initial structure parameters of CNNSCAbase are shown in Table 2(CNNSCAbase Configuration).

Here we first verify and analyze the model training effect of CNNSCAbase with and without SE module. Remove the SE module from the CNNSCAbase model, all other parameters remain unchanged, and name this model CNNSCAnoSE. Train the models CNNSCAnoSE and CNNSCAbase on the training set of the ASCAD dataset with known masks. The training results of the two models are shown in Figure 5(Training effect of CNNSCAnoSE model and CNNSCAbase model):

As shown in Figure 5, when the training iteration reaches 28 times, the accuracy of CNNSCAbase is significantly higher than that of CNNSCAnoSE, which is about $96 \%$. Continue to train the CNNSCAnoSE model, and when the training iteration reaches 70 times, its accuracy rate rises to about $90 \%$. In addition, when the training accuracy of the two models is close, the training time of the 28-iteration CNNSCAbase model is about 1393 seconds, which is significantly less than the training time of the 70-iteration CNNSCAnoSE model, the training time of the latter is about 2240 seconds. This proves that the SE module can promote the improvement of the classification performance of the CNNSCAbase model and can reduce the model training time.

Next, we discuss the hyperparameter optimization of the CNNSCA model. Model structure parameters and training parameters are hyperparameters and need to be set in advance. Later, we will design a set of experimental procedures to optimize these hyperparameters in specific application scenarios. For example, we choose to determine the model parameters first rather than the global training parameters, first determine the number of Conv layers, rather than first determine the kernel size or the number of filters . The reason for this design is: currently, Python's deep learning architecture library ${ }^{[25-26]}$ is mainly used to program the CNN network. When using these library methods, the CNN network structure is usually programmed first. The order in which these parameters appear in the program code will be affected by the library methods, and then the training parameters are designed according to the size of the network and the size of the training set. It is precisely in consideration of the order in which the parameters 
434 appear during programming, we have designed the order of the following experimental 435 procedures.

436

437

438

439

440

441

442

443

444

445

446

447

448

449

450

451

452

453

454

455

456

457

458

459

460

461

462

463

464

465

466

467

468

469

470

471

472

473

2 Selection and optimization of CNN structure parameters for side-channel cryptanalysis 2.1 Structural parameter selection rules

In section Methods 1, the base model CNNSCAbase is set, and the best model after parameter optimization will be named CNNSCAnew later. In CNNSCAbase, in addition to the specific set of structural parameters, the remaining structural parameters need to be customized. These structural parameters include classification function, loss function, optimizer, the number of convolutional layers in each convolution block, the number of convolution kernels in the convolution layer, convolution kernel size, pooling layer pooling mode. When choosing these custom structure parameters, you need to follow the classic rules of building a deep learning network structure ${ }^{[20,22]}$, which can reduce the number of unnecessary test parameters. The rules are as follows:

Rule 1: Set the same parameters for the convolutional layers in the same convolutional block to keep the amount of data generated by different layers unchanged.

Rule 2: The dimensionality of each pooling window is 2 , and the window sliding step is also 2 , each operation reduces the dimensionality of the input data to half.

Rule 3: In the convolutional layer of the $\mathrm{i}$-th block (starting from $\mathrm{i}=1$ ), the number of convolution kernels is $\mathrm{n}: n_{i}=n_{1} \times 2^{i-1}, \mathrm{i} \geqslant 2$. This rule keeps the amount of data processed by different convolution blocks as constant as possible. The network structure characteristics of VGG-16 in this reference [21] are formulated.

Rule 4: The size of the convolution kernel of all convolution layers is the same.

\subsection{Structural parameter optimization}

Among the custom structure parameters, the structure parameters that need to be further adjusted through experimental analysis are: the number of convolution layers in each convolution block, the number of convolution kernels in the convolution layer, the size of the convolution kernel, the pooling mode of the pooling layer, SE module. The experimental process of structural parameter optimization is as follows:

\section{1) Number of convolutional layers}

In Section Methods 1, in the initial CNNSCAbase structure, the number of convolutional layers for each convolution block is 1 , and the convolutional structure is named Cnov1. Refer to the number of convolutional layers of different convolutional blocks of the Alexnet and VGGnet16 prototypes. It is found that the minimum number is 1 and the maximum is 3 , and the small number is distributed in the front convolution block, and the large number is at the back. This is also to build deep learning The common habit of the Internet. Therefore, the upper limit of the number of convolutional layers of the CNNSCAbase convolution block is set to 3, and the 
474 baseline is Cnov1, and a certain convolutional layer parameter configuration can be obtained

475 through two sets of necessary experiments. When training the CNNSCAbase model, the training 476 iteration and batch parameters of the current optimal CNNSCA mode ${ }^{[12]}$ are used, which are 75

477 and 200 (in all experiments in section Methods 2.2, unless otherwise specified, the iteration and 478 batch parameters are used. experiment).

479

480

481

482

483

484

485

486

487

488

489

490

491

492

493

494

495

496

497

498

499

500

501

502

503

504

Experiment 1: Set up a model in which the number of convolutional layers in 5 convolutional blocks is 2, and other parameters are consistent with CNNSCAbase, and the structure is named Cnov2. Then set the number of convolutional layers of the first 4 convolutional blocks to 2 , and the convolutional layer of the last convolutional block to 3. Other parameters are consistent with CNNSCAbase, and the structure is named Cnov3. The specific settings of the number of convolutional layers of each convolution block of Cnov1 3 are shown in Table

3(CNNSCAbase.Conv1-7 Configuration). The three structures constructed are trained and tested, and the results of experiment 1 are shown in Figure 6(Convergence of guessing entropy of Cnov1 3).

From the results in Figure 6, it is found that when Cnov2 and Cnov1 attack the 750th energy trace, their guessing entropy basically converges to 0 , while Cnov 3 cannot converge in a finite number of (1000) attacks. When doing further analysis, if you set two or more convolutional blocks with 3 convolutional layers in the 5 convolutional blocks of the model, the calculation amount and parameter amount of model training will increase by several times, and the 8G GPU memory used in the experiment will be directly exhausted, unable to run the code, then this parameter setting method will have no practical significance. Therefore, the upper limit of the number of convolutional layers for each convolutional block is determined to be 2 .

Experiment 2: On the basis of the conclusion of Experiment 1, the convolutional layer parameter setting of each convolution block is further accurate. As shown in Figure 6, the convergence of the orange line representing the entropy of Cnov2's guess is more stable than that of Cnov1, but it is obvious that there are more convolutional layers, which means that the amount of model calculations and parameters are relatively large, which affects the overall performance of the model. Therefore, four structures of Cnov4 7 are set, and each structure sequentially reduces the

505 number of convolutional layers in each convolution block of Cnov2 by one. The specific settings

506 of the number of convolutional layers of each convolution block of Cnov4 7 are shown in Table

\section{7} 3(CNNSCAbase.Conv1-7 Configuration). Train and test these constructed structures, and the

508 results of experiment 2 are shown in Figure 7(Convergence of guessing entropy of

509 Cnov1 2,4 7).

510

511 The red curve representing the entropy of Cnov5 guessing in Figure 7 converges optimally. 512 Finally, the number of convolutional layers of the Cnov5 structure is determined, and the 513 benchmark is set for the number of convolutional layers of CNNSCAnew. 
514

515

516

517

518

519

520

521

522

523

524

525

526

527

528

529

530

531

532

533

534

535

536

537

538

539

540

541

542

543

544

545

546

547

548

549

550

551

552

553

2) The number of convolution kernels in the convolution layer

It is known that the number of convolution kernels of each convolutional layer of CNNSCAbase is initially set according to Rule 3 . The number of convolution kernels of the first convolutional layer is 64 . Usually increasing the number of convolution kernels means that more dimensional feature extraction is performed on the input data, thereby improving the classification efficiency of the convolutional network. But it will inevitably lead to an increase in the amount of calculation and storage of the attack device, which will lead to an increase in the training time of the model. Therefore, under the condition that the efficiency loss of the guarantee model is not large, the model training time can be reduced by reducing the number of convolution kernels. Since the number of convolution kernels in the later layer increases by a factor of 2 of the number of convolution kernels in the first convolution layer, to determine the number of convolution kernels as a benchmark, it is only necessary to test the number of convolution kernels in the first convolution layer. At the same time, the CNNSCA model in reference [12], the upper limit of the number of convolution kernels in the convolution layer is 512, which can achieve the effect of breaking the density, so the paper also adjusts the upper limit of the number of convolution kernels to 512 .

Experiment 3: Name the four structures tested as filter1, filter2, filter3, and filter4. The convolution kernel values of the first convolution layer are $8,16,32,64$, and the number of convolution kernels of the remaining four convolution blocks is also increased by a factor of 2 respectively. The upper limit of the number of convolution kernels is always 512 . Other structural parameters are the parameters of the current CNNSCAnew. Train and test the filter1 4 structure, and the result of experiment 3 is shown in Figure 8(Convergence of guessing entropy of filter1 4 (epochs=75)).

Figure 8 shows that after 75 iterations of training, the guessing entropy of the filter 4 structure cannot converge. Although the guessing entropy of the filter $1 \sim 3$ structure converges, it fluctuates all the time. When checking the training accuracy of the filter $1 \sim 3$ structure, it is found that the accuracy of the three structures has reached more than $99 \%$, or even reached 1 . Obviously, the model has an overfitting phenomenon, which is the most common problem in neural networks. Therefore, the number of training iterations of the filter $1 \sim 3$ structure is reduced to 40 , the three structures are retrained, and then the test set is attacked again, and the result shown in Figure 9 (Convergence of guessing entropy of filter1 3 (epochs=40)) is obtained. The guessing entropy of the filter1 3 structure in Figure 9 all converge to rank 0 , and filter3 converges to the position of rank 0 earliest. In summary, the benchmark for selecting convolution kernel parameters is the filter3 structure, and the convolution kernel parameters of the CNNSCAnew structure are updated synchronously.

3) Pooling mode of pooling layer

Peer] Comput. Sci. reviewing PDF | (CS-2021:09:65519:1:1:NEW 20 Nov 2021) 
554 It is known that the initial setting mode of the pooling layer of CNNSCAbase is AveragePool,

555

556

557

558

559

560

561

562

563

564

565

566

567

568

569

570

571

572

573

574

575

576

577

578

579

580

581

582

583

584

585

586

587

588

589

590

591

592

593

and another common pooling mode is MaxPooling. According to rule 3, both the pooling window and the pooling step size are still selected here.

Experiment 4: Will test the impact of two pooling modes AveragePool and MaxPooling on the current CNNSCAnew structure. The result of experiment 4 is shown in Figure 10(Convergence of guessing entropy of AveragePool and MaxPool structure).

In Figure 10, it is obvious that the guessing entropy convergence of the average pooling structure is better than the maximum pooling structure, so the benchmark of the pooling layer pooling mode is average pooling, and the pooling mode of the CNNSCAnew structure is set to average pooling.

\section{4) Convolution kernel size}

The size of the convolution kernel of each convolution layer in CNNSCAbase is initially set to $1 \times 3$, or 3 for short. In deep learning, people often reduce the size of the convolution kernel by increasing the depth of the network, thereby reducing the computational complexity of the network. In the VGG-CNNSCA structure, the convolution kernel uses a larger size of 11, and in the Alex-CNNSCA structure, a small size of 3 is used.

Experiment 5: Test the attack effects of the models with the convolution kernel sizes of 3, 5, 7, 9, and 11, and name these five structures as kernel3, kernel5, kernel7, kernel9, and kernel11. The other parameters of these structures are compared with The current CNNSCAnew is the same. The result of experiment 5 is shown in Figure 11(Convergence of guessing entropy of different convolution kernel size structures).

In Figure 11, the convolution kernel size of the structure kernel3 is 3, which guesses that the entropy convergence is better than other structures, so the size 3 is used as the setting reference for the convolution kernel size. At the same time, the size of the convolution kernel of the CNNSCAnew structure is set to 3 .

5) SE module

The attention mechanism in deep learning is essentially similar to the selective visual attention mechanism of humans, and the core role is to select information that is more critical to the current task goal from a large number of information ${ }^{[24]}$. The paper has initially added an SE fixed module to the last four convolution blocks of CNNSCAbase. The initial setting of the dimensional change ratio of the first full link layer of the SE module is $1 / 16$, but this conventional setting is in the SCA scene The suitability of the medium requires further verification.

Peer) Comput. Sci. reviewing PDF | (CS-2021:09:65519:1:1:NEW 20 Nov 2021) 
594 Experiment 6: Test the SE module of the model. The rate of the dimensional change of the first 595 full link layer is $1 / 4,1 / 8,1 / 16$, and $1 / 32$ respectively. The other parameters of the test model are 596 the same as CNNSCAnew. . Experiment 7: Test the attack effect of the SE module used 1, 2, and 5973 times for the last four convolutional blocks in the current CNNSCAnew structure. The results 598 of experiment 6 are shown in Figure 12(Convergence of guessing entropy for different SE 599 dimension ratios).

600

601

602

603

604

605

606

607

608

609

610

611

612

613

614

615

616

617

618

619

620

621

622

623

624

625

626

627

628

629

630

631

632
As shown in Figure 12, when the dimensional ratio of the SE module is $1 / 8$, the guessing entropy convergence of the overall structure of the $\mathrm{CNN}$ is the best. On the basis of this dimensional ratio, the result of Experiment 7 is shown in Figure 13(Convergence of guessing entropy for different number of SE cycles). It is found that when the last four convolution blocks of CNNSCAnew use the SE module twice, the guessing entropy converges fastest. Therefore, the dimensional ratio of the $\mathrm{SE}$ module is $1 / 8$ and the $\mathrm{SE}$ module is looped twice as a new benchmark for the parameters of the SE module in the CNNSCAnew structure.

6) Number of channels at the full link layer

The CNNSCA model in literature $[12,16]$ uses 4096 channels in the fully connected layer, which is similar to the number of channels in the fully connected layer of the original VGGnet and Alexnet structures. Considering that the classification task of the ImageNet competition is 1000 classifications, and only 256 classifications are needed in the SCA scene, the number of channels can be adjusted appropriately to reduce the training complexity of the model.

Experiment 8: Will test the model attack effect of the four cases where the number of channels in the fully connected layer is $4096,3072,2048$, and 1024 . The other parameters of these test models are the same as CNNSCAnew. The reason why the number of channels is not set lower than 1024 is that from the convolutional layer to the fully connected layer, if the vector dimension changes sharply, the feature points of the vector are greatly reduced, which will affect the training effect of the model. The result of experiment 8 is shown in Figure 14(Convergence of guessing entropy of the four channel number structure of FC layer).

It is found from Figure 14 that when the number of channels of the FC layer is 1024, the guessing entropy of its structure converges fastest and continues to be stable. Therefore, 1024 is selected as the reference for the number of channels in the FC layer of the CNNSCAnew structure.

In summary, the parameter benchmark of the CNNSCAnew structure has been optimized. The new structure parameters are shown in Table 4 (CNNSCAnew Configuration).

3 Selection and optimization of CNN training parameters for side-channel cryptanalysis 
633 Almost all experiments in Section Methods 2.2 use the three parameters of 75 iterations, 200

634 batches of learning, and $1 \times 10-4$ learning rate for experiments. These training parameters have

635 little effect on the experimental effects of optimizing various structural parameters, but It is not

636 the optimal setting. The convolutional network of deep learning is applied to the side-channel

637 attack, and these training parameters should also be tuned according to the actual processed side-

638 channel signal data. The order of training parameter tuning is usually learning rate, batch

639 learning amount, and number of iterations ${ }^{[39-40]}$. In the experiment of training parameter

640 optimization, the current CNNSCAnew structure is used. The training parameter optimization

641 experiment process is as follows:

642

643 1) Learning rate

644 The learning rate is a hyperparameter that is artificially set. The learning rate is used to adjust the

645 size of the weight change, thereby adjusting the speed of model training. The learning rate is

646 generally between $0-1$. The learning rate is too large, and the learning will be accelerated in the

647 early stage of model training, making it easier for the model to approach the local or global

648 optimal solution, but there will be large fluctuations in the later stage of the training, and even

649 the value of the loss function may hover around the minimum value, which is always difficult to

650 reach Optimal solution; the learning rate is too small, the model weight adjustment is too slow,

651 and the number of iterations is too much.

652

653 Experiment 9: Will test the impact of five commonly used learning rates on the model's

654 cryptanalysis effect, namely $\operatorname{lr} 1=1 \times 10^{-2}, \operatorname{lr} 2=1 \times 10^{-3}, \operatorname{lr} 3=1 \times 10^{-4}, \operatorname{lr} 4=1 \times 10^{-5}, \operatorname{lr} 5=1 \times 10^{-6}$. The

655 result of experiment 9 is shown in Figure 15(Convergence of model guessing entropy under five 656 learning rates).

657

658 Figure 15 reflects that when the learning rate is $1 \mathrm{r} 2$, the guessing entropy of CNNSCAnew

659 converges fastest and is the most stable. Therefore, $1 \times 10^{-3}$ is selected as the learning rate

660 benchmark of the CNNSCAnew structure.

661

662 2) Batch size

663 The appropriate batch size is more important for the optimization of the model. This parameter

664 does not need to be fine-tuned, just take a rough number, usually $2^{\text {n }}$ (GPU can play a better

665 performance for batches of the power of 2). A batch size that is too large will be limited by the

666 GPU memory, the calculation speed will be slow, and it cannot increase indefinitely (the training

667 set has 50,000 data); it cannot be too small, which may cause the algorithm to fail to converge.

668

669 Experiment 10: According to the size of the ASCAD data set in Section Materials 4, this

670 experiment selects the batch size values: $32,64,128$, and 256 for the experiment. The result of

671 experiment 10 is shown in Figure 16(Convergence of model guessing entropy under four

672 batches). 
673

674 It can be seen from Figure 16 that when the batch learning amount is 128, the guessing entropy

675 of CNNSCAnew converges fastest and is the most stable. Therefore, 128 is selected as the batch

676 size benchmark of CNNSCAnew structure.

677

678 3) Number of iterations (epoch)

679 The number of iterations is related to the fitting performance of the CNNSCA model. The model

680 has been fitted (the accuracy rate reaches 1), and there is no need to continue training; on the

681 contrary, if all epochs have been calculated, but the loss value of the model is still declining, and

682 the model is still optimizing, then the epoch is too small. Should increase. At the same time, the

683 number of iterations of model training also refers to the actual cryptanalysis effect of the model,

684 which is measured by guessing entropy.

685

686

Experiment 11: In experiments 1-10, almost all use iteration number 75 to train the CNNSCA

687 model. This experiment will center on iteration number 75, and train the CNNSCAnew model at

688

689

690

691

692

693

694

695

696

697

698

699

700

701

702

703

704

705

706

707

708

709

710

711

712 10 intervals in the upper and lower intervals to further optimize the model parameter epoch. The interval number of 10 is chosen because the step interval is too small, and the error loss of model training is not much different, so the setting is meaningless; the interval is too large, and repeated experiments may be required to determine an appropriate number of iterations. Therefore, Experiment 11 will test 8 iteration parameters epoch $1=15$, epoch2 $=25$, epoch $3=35$, epoch $4=45$, epoch $5=55$, epoch $6=65$, epoch $7=75$, epoch $8=85$. The current CNNSCAnew structure has achieved higher training accuracy and breaking performance, in order to reduce model calculation pressure and calculation time, lower iteration parameters are usually selected when the model performs better. Therefore, the upper limit of the epoch test parameter is set to 85 . The result of experiment 11 is shown in Figure 17(Convergence of model guessing entropy under eight epochs).

From the results in Figure 17, it is found that the model of epoch1-4 guesses that the entropy does not converge. Separately recalculate the graph of epoch5-8 model. The result is shown in Figure 18(Convergence of model guessing entropy under four epochs). It can be clearly seen that the epoch5-8 model has a convergence trend. Among them, the epoch5 curve is closest to the position of ranking 0 , and the epoch8 curve first converges to ranking 0 , but afterwards it fluctuates more widely, and it is obviously over-fitting. The convergence of epoch 6 and 7 is similar, the curve begins to fluctuate greatly, and it is close to ranking 0 in the later period.

Experiment 12: Continue to debug the epoch parameters in a smaller range, and test the other two iterations with an interval of only 5 : epoch $60=60$, epoch $70=70$. The trained epoch 60 and epoch70 models and the previously trained epoch5, epoch6, and epoch7 models are simultaneously attacked on the target set. The results of Experiment 12 are shown in Figure 19(Convergence of model guessing entropy under five epochs). 
713

714 Figure 19 shows that the guessed entropy of the epoch70 model converges best, and its guessed

715 entropy converges fastest and is the most stable. Therefore, 70 is selected as the training iteration

716 benchmark of the CNNSCAnew structure.

717

718 Results

7191 Get a new model CNNSCAnew for attacking ASCAD data set with known first-order mask 720 protection.

721 According to the 12 sets of experiments in Section Methods 2 and Section Methods 3, the best 722 benchmarks for CNNSCAnew structure parameters and training parameters are demonstrated.

723 The CNNSCAnew model contains 5 convolutional blocks, 8 convolutional layers, and 3 fully

724 connected layers. The size of the convolution kernel of each convolution layer is 3 , the activation

725 function is ReLU, and the padding is Same. Each convolutional block is equipped with a pooling

726 layer, the pooling layer selects the average pooling mode, and the pooling window is $(2,2)$. The

727 number of output channels of the convolution layer in the convolution block 1-5 starts from 32

728 and increases by a multiple of 2 in turn. Two SE modules are added after the convolution layer

729 of each convolution block in the convolution block 2-4, and the dimension ratio of the SE is set

730 to $1 / 8$. In the first two fully connected layers, set the number of output channels to 1024 and the

731 activation function to ReLU. The output channel number of the third fully connected layer is the

732 target classification number 256, and the classification function is Soft-max. The global

733 configuration loss function is crossentropy, the optimization method is RMSprop, the number of

734 training iterations is 70 , the learning rate is $1 \times 10^{-3}$, and the batch learning volume is 128 . All

735 parameters of the newly obtained CNNSCAnew are shown in Table 5(CNNSCAnew

736 Configuration).

737

7382 The CNNSCA model design method and the convolutional network hyperparameter

739 optimization scheme for side-channel attack are refined.

740 The CNNSCA model design method is refined: comprehensively utilize the advantages of VGG-

741 CNNSCA model classification and fitting efficiency and Alex-CNNSCA model occupy less

742 computing resources, while using SEnet's SE module to reduce the gradient dispersion problem

743 of error back propagation in deep neural networks to save calculation time, a new basic model of

744 CNNSCA was designed, named CNNSCAbase.

745

746 At the same time, the hyperparameter optimization scheme of the convolutional network used for

747 side-channel attacks is refined: design the structural parameter optimization experiment and the

748 training parameter optimization experiment, and use CNNSCAbase to implement the attack

749 training. According to parameter selection rules, common sense of parameter optimization of

$750 \mathrm{CNN}$ model, and data characteristics of actual application scenarios, the test parameters of each

751 experiment are designed, and unnecessary test parameters are excluded. Each time, according to

752 the cryptanalysis results of the experiment, the parameters that make CNNSCAbase's 
753 cryptanalysis effect better are selected. Relying on two sets of experimental processes, a

754 hyperparameter optimization scheme is formed, and the hyperparameters finally determined by

755 the experiment are used as the parameters of the new model CNNSCAnew.

756

\section{Discussion}

758 Comparative analysis of CNNSCAnew and other profiling side-channel attack methods

7591 Comparative analysis of CNNSCAnew, classic template attack and MLPSCA

760 Experiment 13: Compare the cryptanalysis's performance of CNNSCAnew with the HW-based

$761 \mathrm{TA}^{[1]}$ and MLPSCA method proposed by Benadjila et al ${ }^{[12]}$. TA and MLPSCA are the profiling

762 methods that performed better in the early traditional profiling methods and the later new

763 profiling methods, respectively. Experiment 13 carried out an attack on the ASCAD data set with

764 a known mask, which represents the realization of the encryption in an unprotected state. The

765 result of experiment 13 is shown in Figure 20(TA, MLPSCA, CNNSCAnew guessing entropy

766 convergence).

767

768

769

It can be seen from Figure 20 that CNNSCAnew's guessing entropy convergence is significantly better than TA and MLPSCA.

770

7712 Comparative analysis with other existing CNNSCA

772 Experiment 14: Compare the breaking performance of CNNSCAnew model with VGG-

$773 \mathrm{CNNSCA}^{[12]}$ and Alex-CNNSCA ${ }^{[16]}$. The latter two methods are the profiling methods with

774 better performance among the latest profiling methods. Among them, VGG-CNNSCA in [12]

775 uses the ASCAD public data set, and Alex-CNNSCA in [16] uses a self-collected data set.

776 Experiment 14 carried out an attack on the ASCAD data set with a known mask, which

777 represents the realization of the encryption in an unprotected state. The result of experiment 14 is

778 shown in Figure 21(CNNSCAnew, VGG-CNNSCA, Alex-CNNSCA guessing entropy

779 convergence).

780

781

782

783

It can be seen from Figure 21 that the CNNSCAnew proposed in this paper has a better guessing entropy convergence than other CNNSCAs. In [12], the guessing entropy of VGG-CNNSCA

784 requires at least 650 power consumption traces to converge to rank zero, and the model training time takes 37 minutes. The CNNSCAnew method constructed in this paper only requires 61

785 power consumption traces, and the model training time only needs about 28 minutes. The training time of CNNSCAnew and VGG-CNNSCA in this paper are shown in Figure 22(CNNSCAnew and VGG-CNNSCA training time).

788

789

After comparing CNNSCAnew with VGG-CNNSCA and Alex-CNNSCA, the model

790 comparison analysis and the cryptanalysis performance comparison analysis, the results are summarized in Table 6(Comparative analysis of CNNSCAnew, VGG-CNNSCA and Alex-

792 CNNSCA) to show. 


\section{Conclusions}

795

796

797

798

799

800

801

802

803

804

805

806

807

808

809

810

811

812

813

814

815

816

817

818

819

820

821

822

823

824

825

826

827

828

829

830

831

832

Among the profiling side-channel cryptography attack methods, the most popular one is CNNSCA, a side-channel attack method combined with deep learning convolutional neural network algorithms. Its cryptanalysis performance is significantly better than traditional profiling methods. Among the existing CNNSCA methods, the CNNSCA network models that achieve cryptanalysis mainly include CNNSCA based on the VGG variant (VGG-CNNSCA) and CNNSCA based on the Alexnet variant (Alex-CNNSCA). The learning capabilities and cryptanalysis performance of these CNNSCA models it is not optimal. The paper aims to explore effective methods to obtain the performance gains of the new side-channel attack method CNNSCA.

After studying the related knowledge, necessary structure and core algorithm of CNNSCA, the paper found that CNNSCA model design and hyperparameter optimization can be used to improve the overall performance of CNNSCA. In terms of CNNSCA model design, the advantages of VGG-CNNSCA model classification and fitting efficiency and the Alex-CNNSCA model occupying less computing resources can be used to design a new CNNSCA basic model. In order to better reduce the gradient dispersion problem of error back propagation in the deep network, it is a very effective method to embed the SE module in this basic model; in terms of the hyperparameter optimization of the CNNSCA model, the above basic model is applied to side-channel leakage A known first-order mask data set in the public database (ASCAD). In this specific application scenario, according to the model design rules and actual experimental results, unnecessary experimental parameters can be excluded to the greatest extent. Various hyperparameters of the model are optimized within the parameter interval to improve the performance of the new CNNSCA, and the final determination benchmark for each hyperparameter is given. Finally, a new CNNSCA model optimized architecture for attacking unprotected encryption devices is obtained —CNNSCAnew. The paper also verified through experimental comparison that CNNSCAnew's cryptanalysis effect is completely superior to traditional profiling methods and the new profiling methods in literature $[12,16]$. In the literature $[12,16]$, the results of CNNSCA's guessing entropy are: convergence to 650 and oscillation. The result of CNNSCAnew's guessing entropy proposed in this paper is to converge to a minimum of 61. Under the same experimental environment and experimental equipment conditions, literature [12] took 40 minutes from model training to attacking the key, while the total calculation time of CNNSCAnew was shortened to 30 minutes.

It should be noted that in practice, the results of each training of the CNNSCAnew model will have a slight deviation. This is a normal phenomenon during neural network training and will not affect the average performance of the model. While proposing the new CNNSCA method, the paper also provides a more comprehensive and detailed design plan and optimization method for the side-channel cryptanalysis researchers who need to design the CNNSCA model. In the

Peer) Comput. Sci. reviewing PDF | (CS-2021:09:65519:1:1:NEW 20 Nov 2021) 
833 future, we can use these design schemes and optimization methods to continue to explore the 834 CNNSCA model that is more suitable for attacking protected equipment to achieve efficient 835 attacks on encrypted equipment with protection, which is of great significance to information 836 security and encryption protection.

837

\section{Acknowledgements}

839 In the paper, from the topic selection of the paper, the structure of the chapter to the scrutiny of 840 words, I got the careful guidance of my tutor, professor Chen Kaiyan; in life, my teacher is very 841 concerned and caring for me. In addition, I sincerely thank professor Li Xiongwei and Zhang 842 Yang. They put forward many opinions and suggestions on my paper, which greatly inspired the 843 students and opened up a lot of thinking. Thanks to the classmates and friends around me. They 844 gave me encouragement and assistance, and let me feel the warmth of this big family. May our

845 friendship last forever. Thanks to the university for providing me with a good learning platform, 846 which gave me a new start.

847

\section{References}

849 [1] Mangard S, Oswald E, Popp T. Energy analysis attack [M]. Translated by Feng Dengguo, 850 Zhou Yongbin, and Liu Jiye. Beijing: Science Press, 2010.

851 [2] Kocher P, Jaffe J, Jun B. Differential power analysis[C]. Annual International Cryptology 852 Conference. Springer, Berlin, Heidelberg, 1999: 388-397.

853 [3] Brier E, Clavier C, Olivier F. Correlation power analysis with a leakage

854 model[C].International workshop on cryptographic hardware and embedded systems.

855 Springer,Berlin, Heidelberg, 2004: 16-29.

856 [4] Gierlichs B, Batina L, Tuyls P, Preneel B. Mutual information analysis[C]. International 857 Workshop on Cryptographic Hardware and Embedded Systems. Springer, Berlin,Heidelberg, 858 2008: 426-442.

859 [5] Chari S, Rao J R, Rohatgi P. Template attacks[C]. International Workshop on Cryptographic 860 Hardware and Embedded Systems. Springer, Berlin, Heidelberg, 2002:13-28.

861 [6] Lerman, L., Bontempi, G., Markowitch, O.: Power analysis attack: An approachbased on 862 machine learning. Int. J. Appl. Cryptol. 3(2) (June 2014) 97-115

863 [7] Picek, S., Heuser, A., Guilley, S.: Template attack versus Bayes classifier. Journal of 864 Cryptographic Engineering 7(4) (Nov 2017) 343-351

865 [8] Choudary, O., Kuhn, M.G.: Efficient template attacks. In Francillon, A., Rohatgi,P., eds.: 866 Smart Card Research and Advanced Applications - 12th International Conference, CARDIS 867 2013, Berlin, Germany, November 27-29, 2013. Revised Selected Papers. Volume 8419 of 868 LNCS., Springer (2013) 253-270

869 [9] Lerman, L., Poussier, R., Bontempi, G., Markowitch, O., Standaert, F.: Template Attacks vs. 870 Machine Learning Revisited (and the Curse of Dimensionality in Side-Channel Analysis). In: 871 COSADE 2015, Berlin, Germany, 2015. Revised Selected Papers. (2015) 20-33 
872 [10] Lerman, L., Bontempi, G., Markowitch, O.: A machine learning approach against a masked 873 AES - Reaching the limit of side-channel attacks with a learning model.J. Cryptographic 874 Engineering 5(2) (2015) 123-139

875 [11] Picek, S., Heuser, A., Jovic, A., Legay, A.: Climbing down the hierarchy: Hierarchical 876 classification for machine learning side-channel attacks. In Joye, M., Nitaj,A., eds.: Progress in 877 Cryptology - AFRICACRYPT 2017: 9th International Conference on Cryptology in Africa, 878 Dakar, Senegal, May 24-26, 2017, Proceedings, Cham, Springer International Publishing (2017) $879 \quad 61-78$

880 [12] Benadjila R, Prouff E, Strullu R, Cagli E, Dumas C. Study of deep learning techniques for 881 side-channel analysis and introduction to ASCAD database[J]. ANSSI, France \& CEA,LETI, 882 MINATEC Campus, France.2018, 22: 2018.

883 [13] Maghrebi, H., Portigliatti, T., Prouff, E.: Breaking cryptographic implementations using 884 deep learning techniques. In: Security, Privacy, and Applied Cryptography Engineering 6th 885 International Conference, SPACE 2016, Hyderabad, India, December 14-18, 2016, Proceedings. 886 (2016) 3-26

887 [14] Picek, S., Samiotis, I.P., Heuser, A., Kim, J., Bhasin, S., Legay, A.: On the performance of 888 convolutional neural networks for side-channel analysis. Cryptology ePrint Archive, Report 889 2018/004 (2018) https://eprint.iacr.org/2018/004.

890 [15] Cagli E, Dumas C, Prouff E. Convolutional neural networks with data augmentation against 891 jitter-based countermeasures[C]. International Conference on Cryptographic Hardware and 892 Embedded Systems. Springer, Cham, 2017: 45-68.

893 [16] Guo Dongxin, Chen Kaiyan, Zhang Yang, Hu Xiaoyang, Wei Yanhai. A new method for 894 attacking encrypted chip templates based on Alexnet convolutional neural network. Computer 895 Measurement and Control, 2018. 26(10): Pages 246-249+254.

896 [17] Guo Dongxin, Chen Kaiyan, Zhang Yang, Zhang Xiaoyu, Li Jianlong. A new method of 897 attacking encrypted chip templates based on VGGNet convolutional neural network. Computer 898 Application Research, 2019. 36(09): Page 2809-2812+2855.

899 [18] Kim J, Picek S, Heuser A, Bhasin S, Hanjalic A. Make some noise. unleashing the power of 900 convolutional neural networks for profiled side-channel analysis[J]. IACR Transactions on 901 Cryptographic Hardware and Embedded Systems, 2019: 148-179.

902 [19] Krizhevsky,A.,Sutskever,I.,Hinton,G.E.:Imagenet classification with deep convolutional 903 neural networks. Commun. ACM 60(6),84-90 (2017). https://doi.org/10.1145/3065386

904 [20] Zeiler,M.D.,Fergus,R.:Visualizing and understanding convolutional networks. In:European 905 Conference on Computer Vision,pp.818-833. Springer (2014)

906 [21] Simonyan, K., Zisserman, A.: Very deep convolutional networks for large-scale image 907 recognition (2014). arXiv preprintarXiv:1409.1556

908 [22] Szegedy, C., Liu, W., Jia, Y., Sermanet, P., Reed, S., Anguelov, D.,Erhan, D., Vanhoucke, 909 V., Rabinovich, A.: Going deeper with convolutions. In: Proceedings of the IEEE Conference on 910 Computer Vision and Pattern Recognition, pp. 1-9 (2015) 
911 [23] He,K.,Zhang,X.,Ren,S.,Sun,J.:Deep residual learning for image recognition. In: Proceedings 912 of the IEEE Conference on Computer Vision and Pattern Recognition, pp. 770-778 (2016)

913 [24] Jie H, Li S, Gang S, Albanie S. Squeeze-and-Excitation Networks[J]. IEEE Transactions on 914 Pattern Analysis and Machine Intelligence, 2017.

915 [25] Eldeeb A, Bursztein E, Chollet F, Haifeng Jin, Qianli Scott Zhu.: Keras (2015).

916 https://github.com/fchollet/keras

917 [26] Abadi M, Agarwal A, Barham P, Brevdo E, Chen Z, Citro C, Corrado G.S., Davis A, Dean J, 918 Devin M, Ghemawat S,Goodfellow I, Harp A, Irving G, Isard M, Jia Y, Jozefowicz R, Kaiser L, 919 Kudlur M, Levenberg J, Mané D, Monga R, Moore S, Murray D, Olah C, Schuster M, Shlens J, 920 Steiner B, Sutskever I, Talwar K, Tucker P, Vanhoucke V, Vasudevan V, Viégas F, Vinyals O, 921 Warden P, Wattenberg M, Wicke M, Yu Y, Zheng X.: TensorFlow: Large-scale machine 922 learning on heterogeneous systems (2015). https://www.tensorflow.org/. Software available from 923 tensorflow.org.

924 [27] D. H. Hubel T. N. Wiesel "Receptive Fields And Functional Aechitecture of Monkey Striate 925 Cortex”[J]. Physiol. (1968), 195, pp. 215-243 With 3 plates and 14 text figures Printed in Great 926 Britain.

927 [28] Lecun Y, Bengio Y. Convolutional Networks for Images, Speech, and Time Series[J]. 1998. 928 [29]Russakovsky O, Deng J, Su H, Krause J, Satheesh S, Ma S, Huang Z, Karpathy A, Khosla A, 929 Bernstein M. :Imagenet large scale visual recognition challenge.Int.J.Comput.Vis.115(3),211$930252(2015)$.

931 [30] Kim, T., Lee, J., Nam, J.: Sample-level CNN architectures for music auto-tagging using raw 932 waveforms. In: 2018 IEEE International Conference on Acoustics, Speech and Signal Processing, 933 ICASSP 2018, Calgary, AB, Canada, April 15-20, 2018.(2018) 366-370

934 [31] Choi K, Fazekas G, Sandler M, Cho K. Convolutional Recurrent Neural Networks for 935 Music Classification[J]. IEEE, 2016.

936 [32] Ioffe, S., Szegedy, C.: Batch normalization: accelerating deep network training by reducing 937 internal covariate shift. CoRR (2015).arXiv:1502.03167.

938 [33] Goodfellow, I.J., Bengio, Y., Courville, A.C.: Deep Learning.Adaptive Computation and 939 Machine Learning. MIT Press, Cambridge (2016).

940 [34] Han Liqun, Kang Qian. Artificial Neural Network Theory, Design and Application941 Nerve Cells, Neural Networks and Neural System[J].Journal of Beijing Technology and 942 Business University: Natural Science Edition, 2005, 23(1): 52-52.

943 [35] Hawkins D M. The problem of overfitting[J]. Journal of chemical information and computer 944 sciences, 2004, 44(1): 1-12.

945 [36] Standaert F X, Malkin T G, Yung M. A unified framework for the analysis of side-channel 946 key recovery attacks[C]. Annual international conference on the theory and applications of 947 cryptographic techniques. Springer, Berlin, Heidelberg, 2009: 443-461.

948 [37] Masure L, Dumas C, Prouff E. A comprehensive study of deep learning for side-channel 949 analysis[J]. IACR Transactions on Cryptographic Hardware and Embedded Systems, 2020: 348950375. 
951 [38] Lecun Y, Cortes C. The mnist database of handwritten digits[J].

952 http://www.research.att.com/ yann/ocr/mnist/, 2010.

953 [39] Smith S L, Kindermans P J, Ying C, Le Q V. Don't Decay the Learning Rate, Increase the 954 Batch Size[J]. 2017.

955 [40] Soltanolkotabi M, Javanmard A, Lee J D. Theoretical insights into the optimization

956 landscape of over-parameterized shallow neural networks[J]. IEEE Transactions on Information 957 Theory, 2017, PP(2):742-769. 
Figure 1

SEnet module 


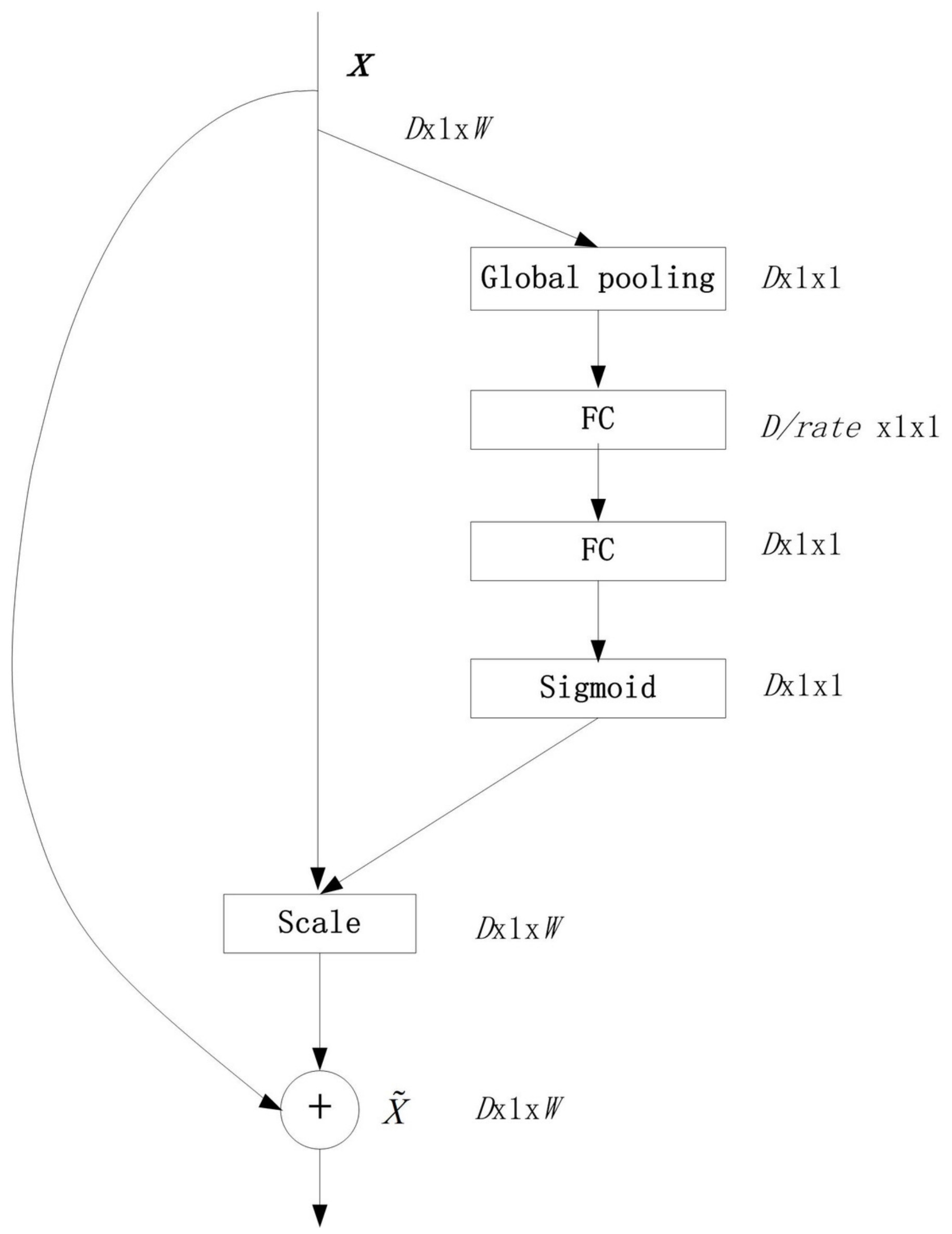


Figure 2

Convolution_calculation_process

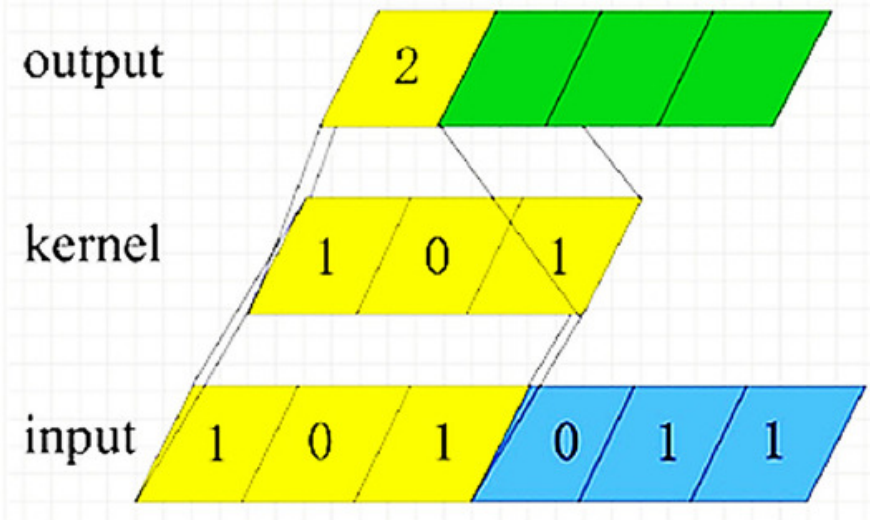

(a)

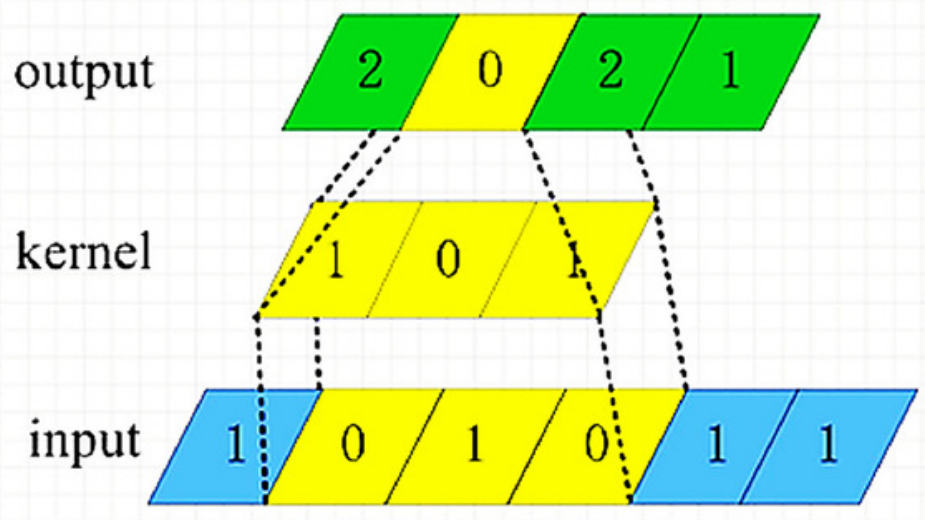

(b) 
Figure 3

Pooling_calculation_process

input

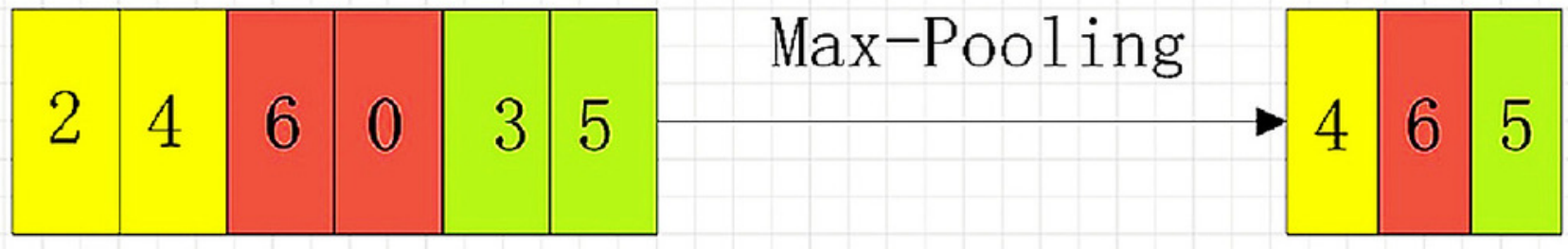

(a)

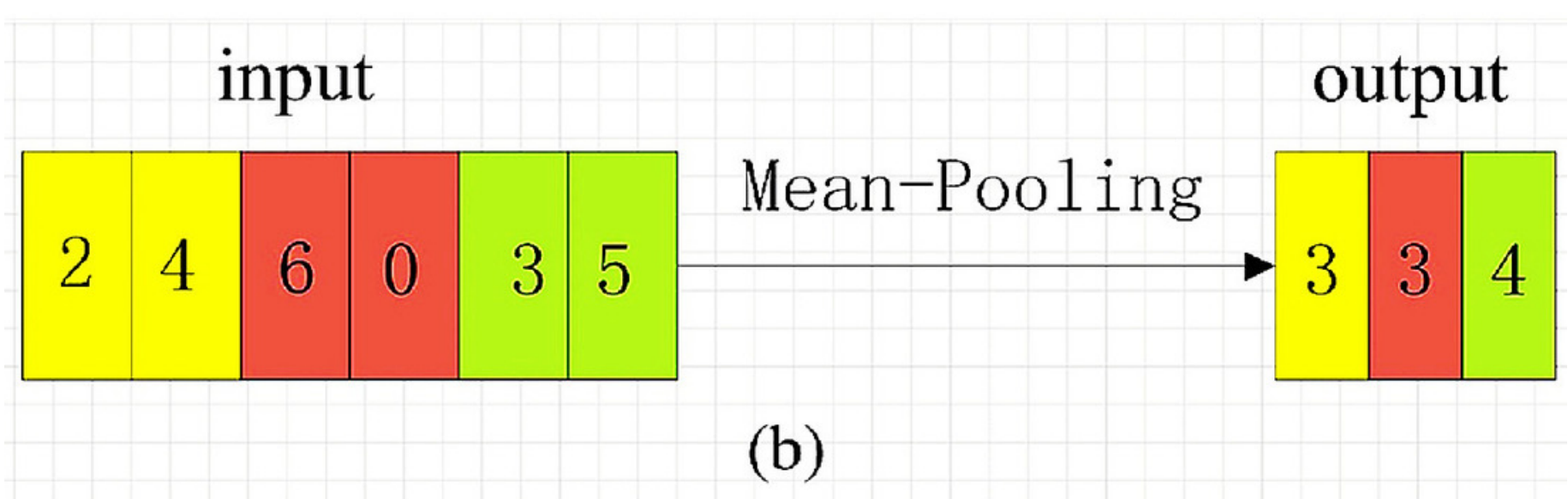


Figure 4

\section{Convolutional network structure in a side-channel attack scenario}

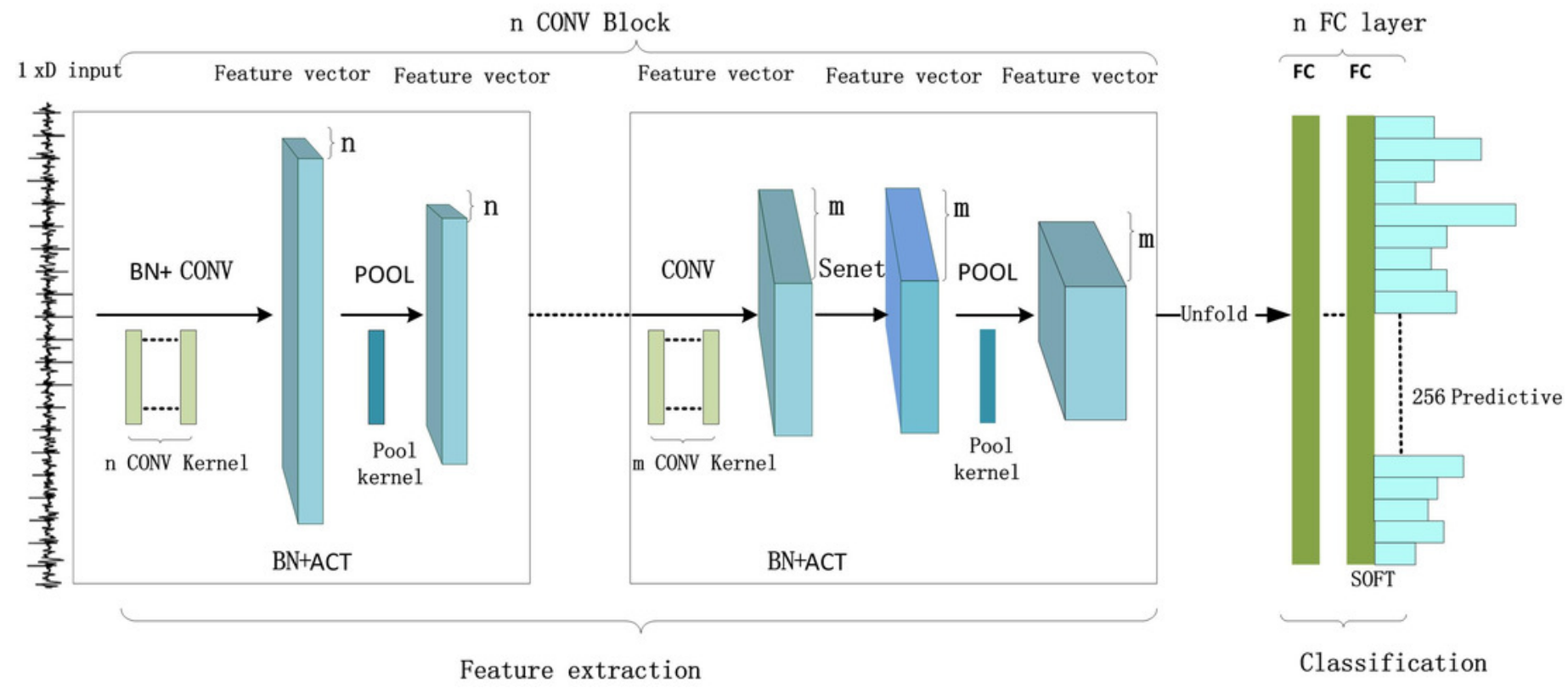




\section{Figure 5}

Training_effect_of_CNNSCAnoSE_model_and_CNNSCAbase_model

\begin{tabular}{|c|c|c|c|}
\hline $\begin{array}{l}\text { CNNSCAbase } \\
\text { (28 epochs) }\end{array}$ & 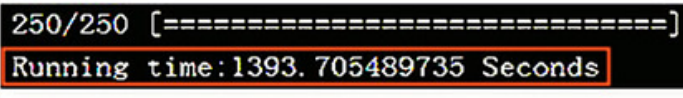 & - 57s 227ms/step - loss: $0.6544-$ & accuracy: 0.9666 \\
\hline $\begin{array}{l}\text { NNSCAnoSE } \\
\text { (28 epochs) }\end{array}$ & 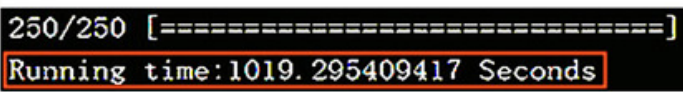 & & \\
\hline $\begin{array}{l}\text { NNSCAno } \\
\text { (70 epochs) }\end{array}$ & 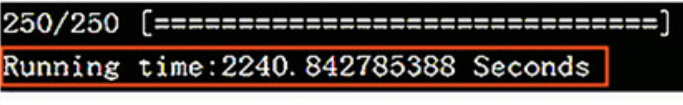 & & \\
\hline
\end{tabular}




\section{Figure 6}

Convergence_of_guessing_entropy_of_Cnov1 3

Each curve represents the convergence trend of guessing entropy under the six model structures of Cnov1 3. The abscissa represents the number of energy traces used in the attack, and the ordinate represents the ranking of the guessing entropy.

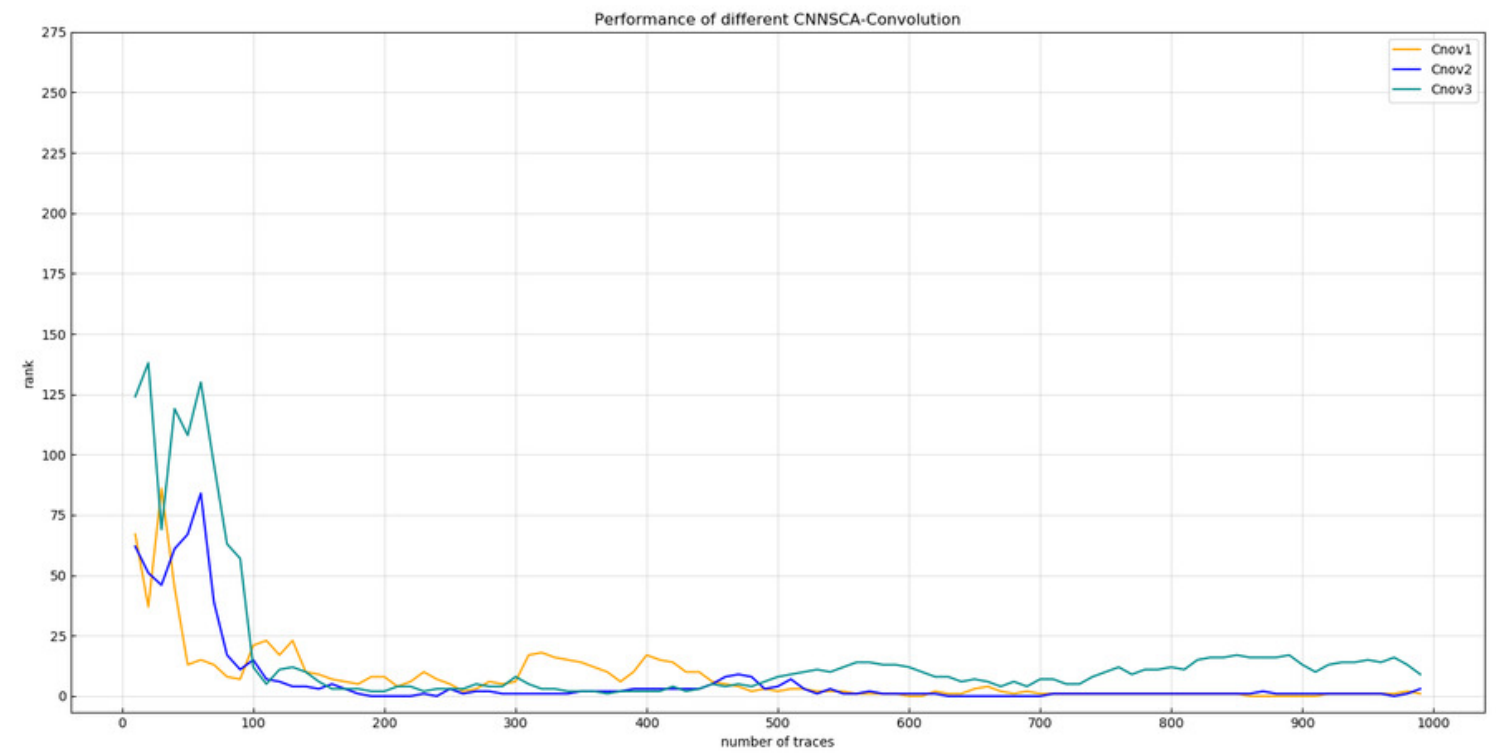


Figure 7

Convergence_of_guessing_entropy_of_Cnov1 2,4 7

Each curve represents the convergence trend of guessing entropy under the six model structures of Cnov1 2,4 7. The abscissa represents the number of energy traces used in the attack, and the ordinate represents the ranking of the guessing entropy.

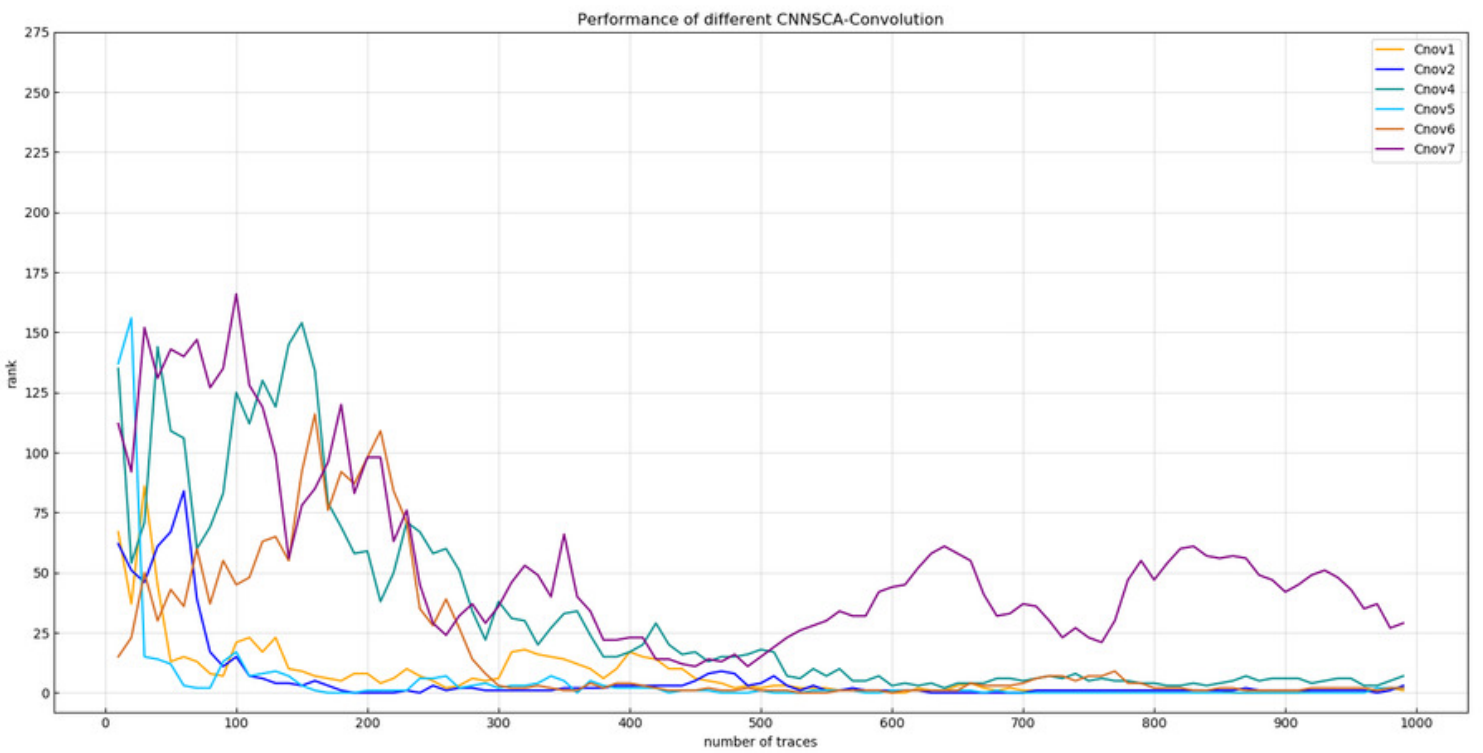


Figure 8

Convergence_of_guessing_entropy_of_filter1 4_(epochs=75)

Each curve represents the model guessing entropy of the four convolution kernel sizes. The abscissa represents the number of energy trajectories used in the attack, and the ordinate represents the order of guessing entropy.

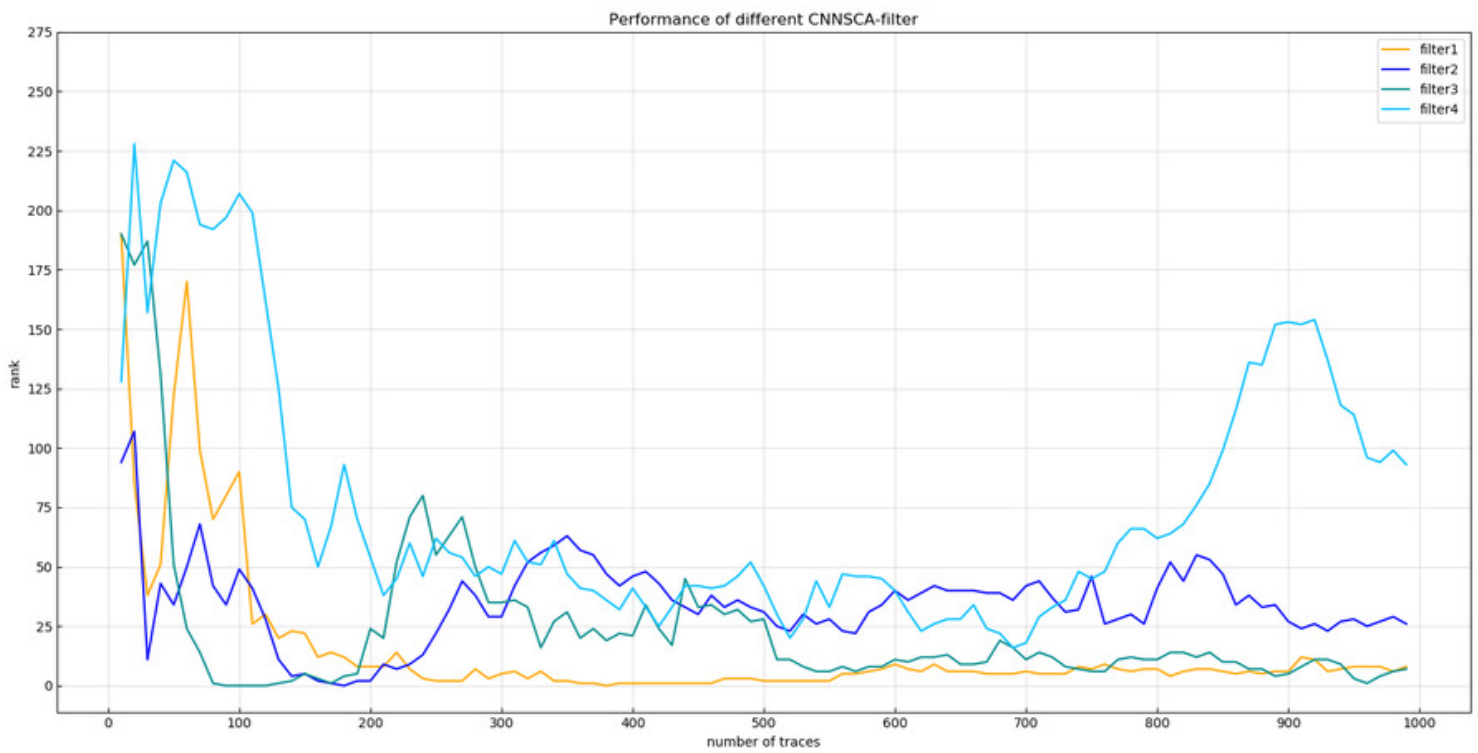


Figure 9

Convergence_of_guessing_entropy_of_filter1 3_(epochs=40)

Each curve represents the model guessing entropy of the three convolution kernel sizes. The abscissa represents the number of energy trajectories used in the attack, and the ordinate represents the order of guessing entropy.

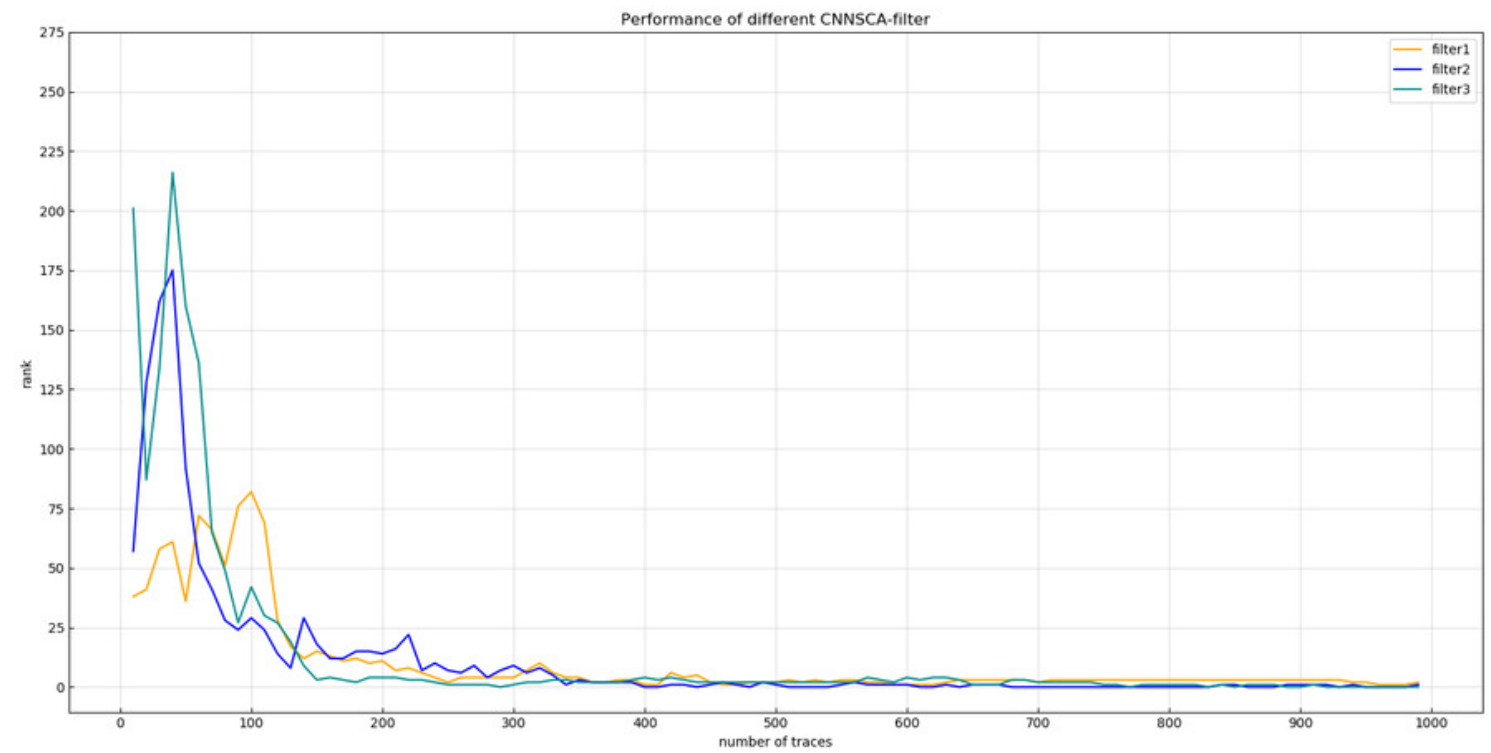




\section{Figure 10}

Convergence_of_guessing_entropy_of_AveragePool_and_MaxPool_structure

Each curve represents the model guessing entropy of two pooling methods. The abscissa represents the number of energy trajectories used in the attack, and the ordinate represents the order of guessing entropy.

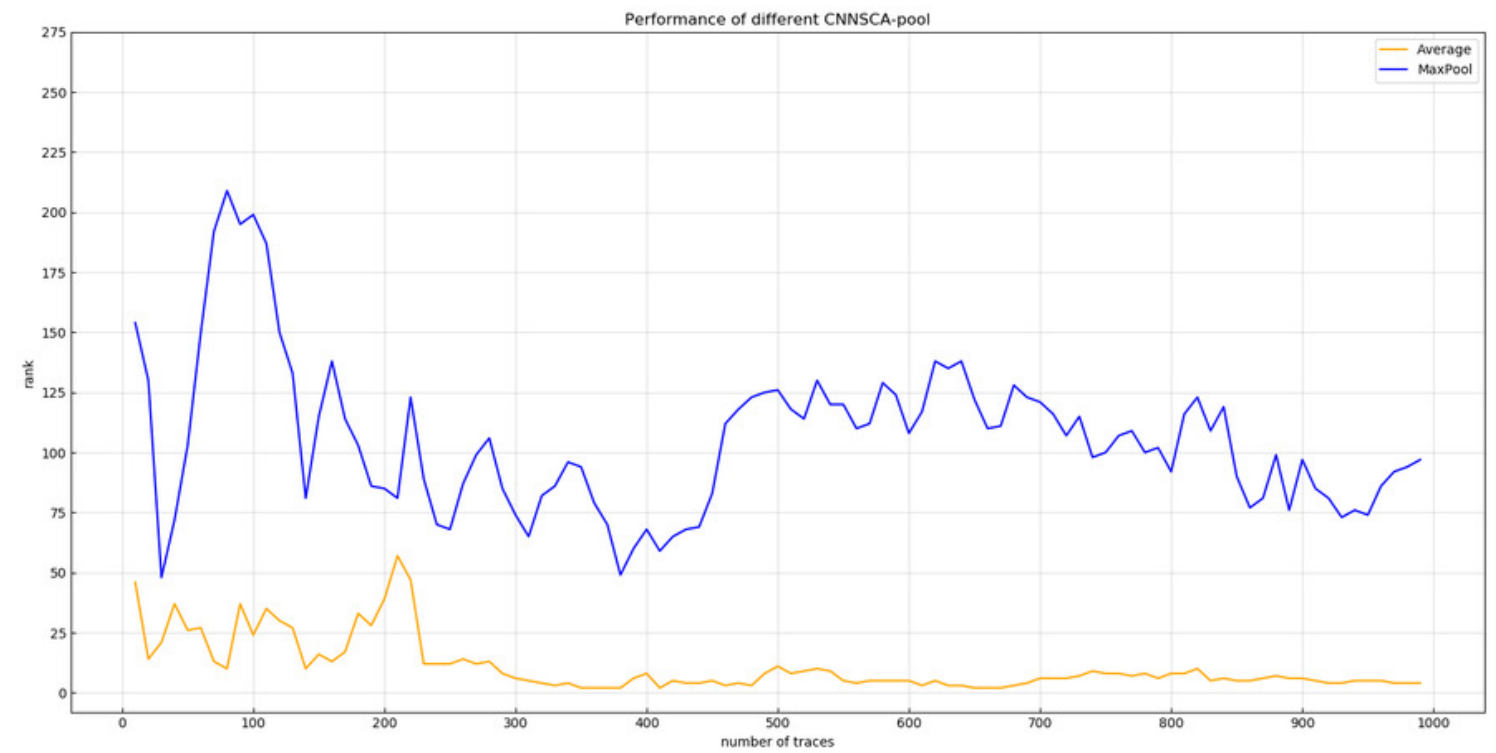




\section{Figure 11}

Convergence_of_guessing_entropy_of_different_convolution_kernel_size_structures

Each curve represents the model guessing entropy of 5 convolution kernel sizes structures.

The abscissa represents the number of energy trajectories used in the attack, and the ordinate represents the order of guessing entropy.

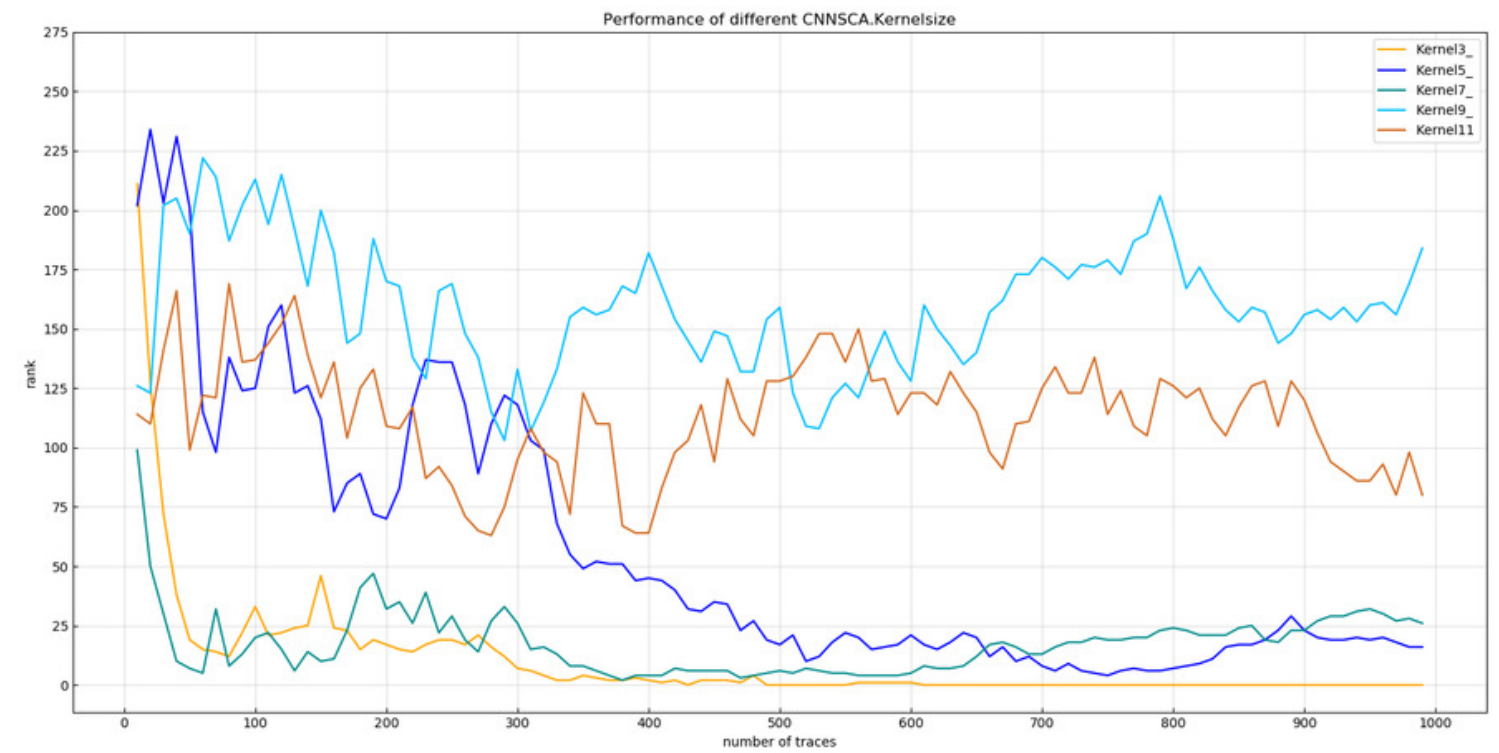




\section{Figure 12}

Convergence_of_guessing_entropy_for_different_SE_dimension_ratios

Each curve represents the model guessing entropy of the four SE dimension ratios. The abscissa represents the number of energy trajectories used in the attack, and the ordinate represents the order of guessing entropy.

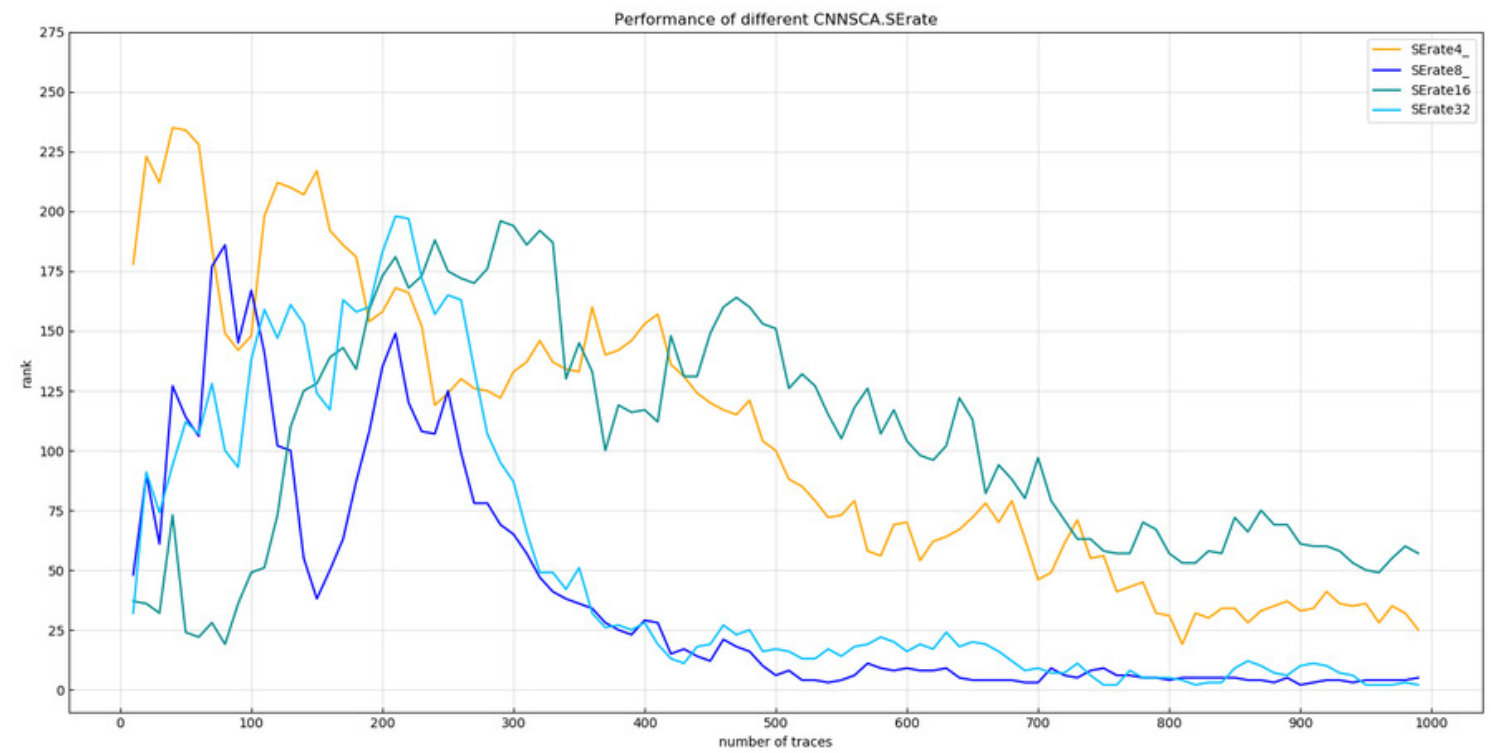




\section{Figure 13}

Convergence_of_guessing_entropy_for_different_number_of_SE_cycles

Each curve represents the model guessing entropy of the three SE cycles. The abscissa represents the number of energy trajectories used in the attack, and the ordinate represents the order of guessing entropy.

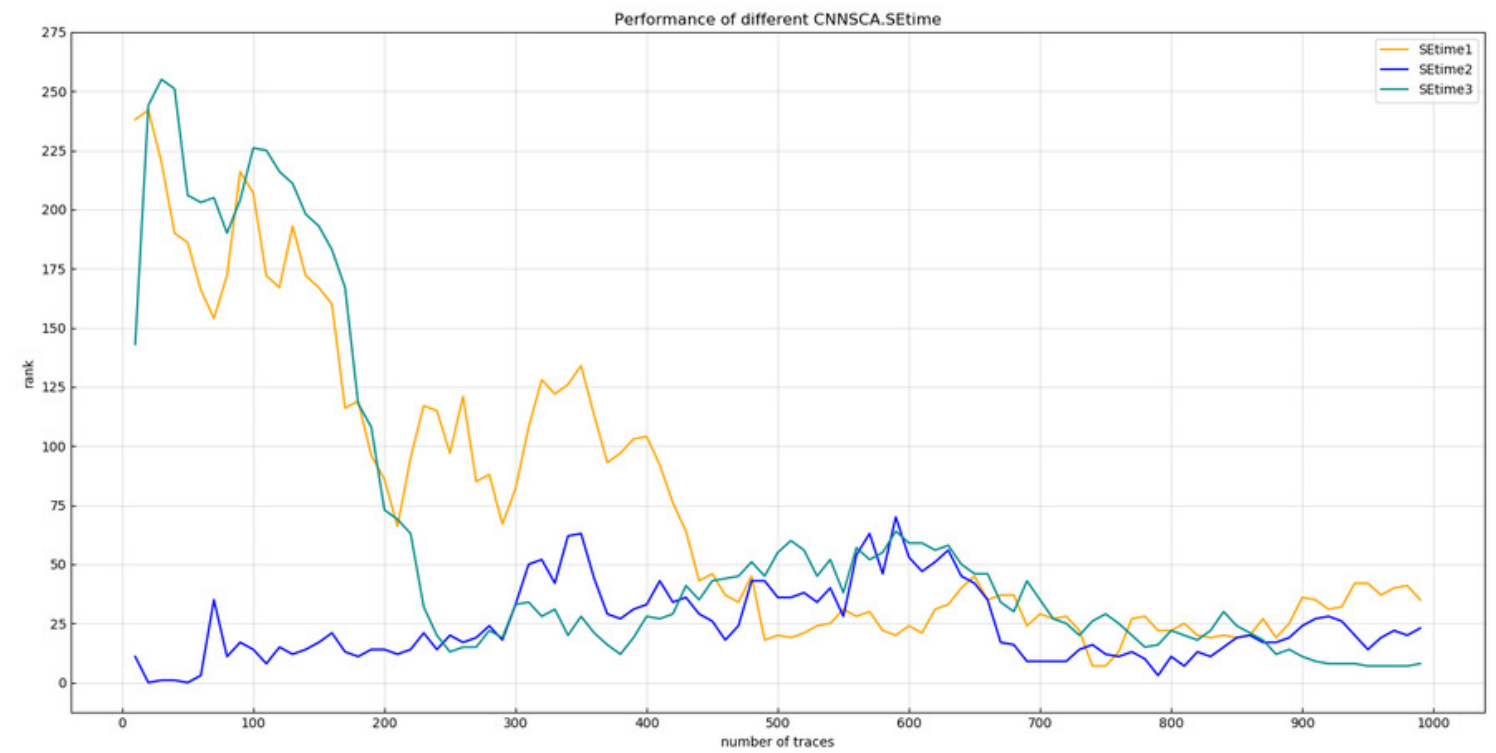




\section{Figure 14}

Convergence_of_guessing_entropy_of_the_four_channel_number_structure_of_FC_layer

Each curve represents the model guessing entropy of the four FC layer structures. The abscissa represents the number of energy trajectories used in the attack, and the ordinate represents the order of guessing entropy.

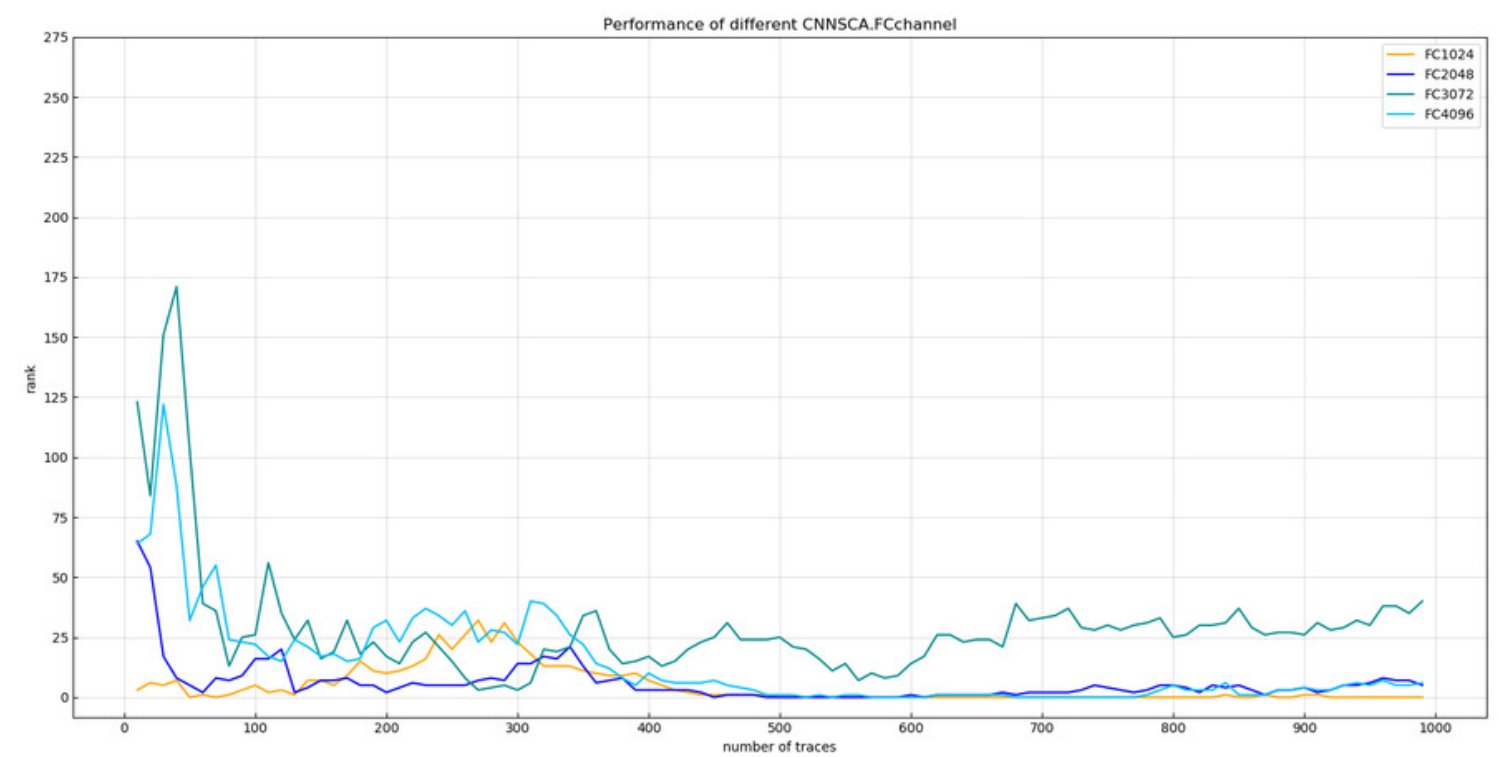




\section{Figure 15}

Convergence_of_model_guessing_entropy_under_five_learning_rates

Each curve represents the model guessing entropy of five learning rates . The abscissa represents the number of energy trajectories used in the attack, and the ordinate represents the order of guessing entropy.

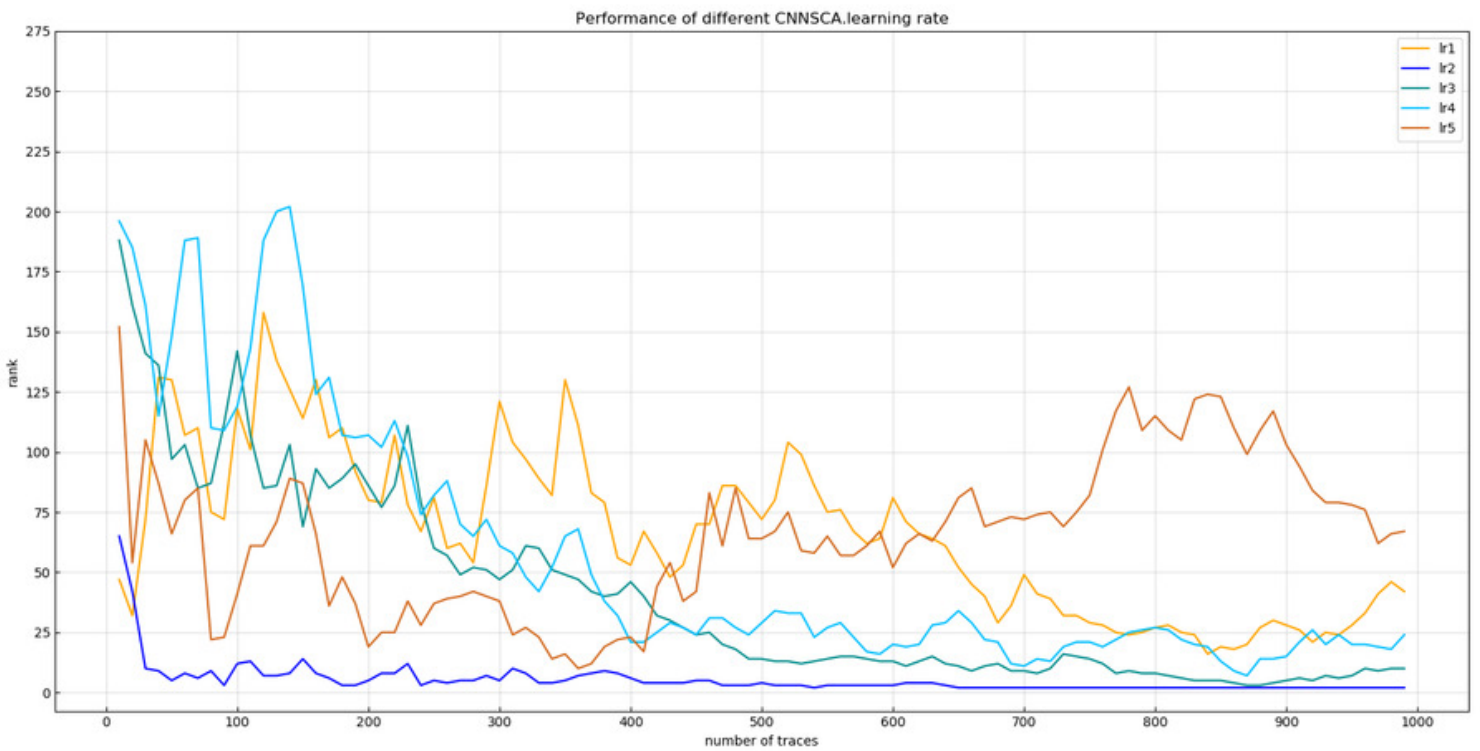


Figure 16

Convergence_of_model_guessing_entropy_under_four_batches

Each curve represents the model guessing entropy of the four training batches. The abscissa represents the number of energy trajectories used in the attack, and the ordinate represents the order of guessing entropy.

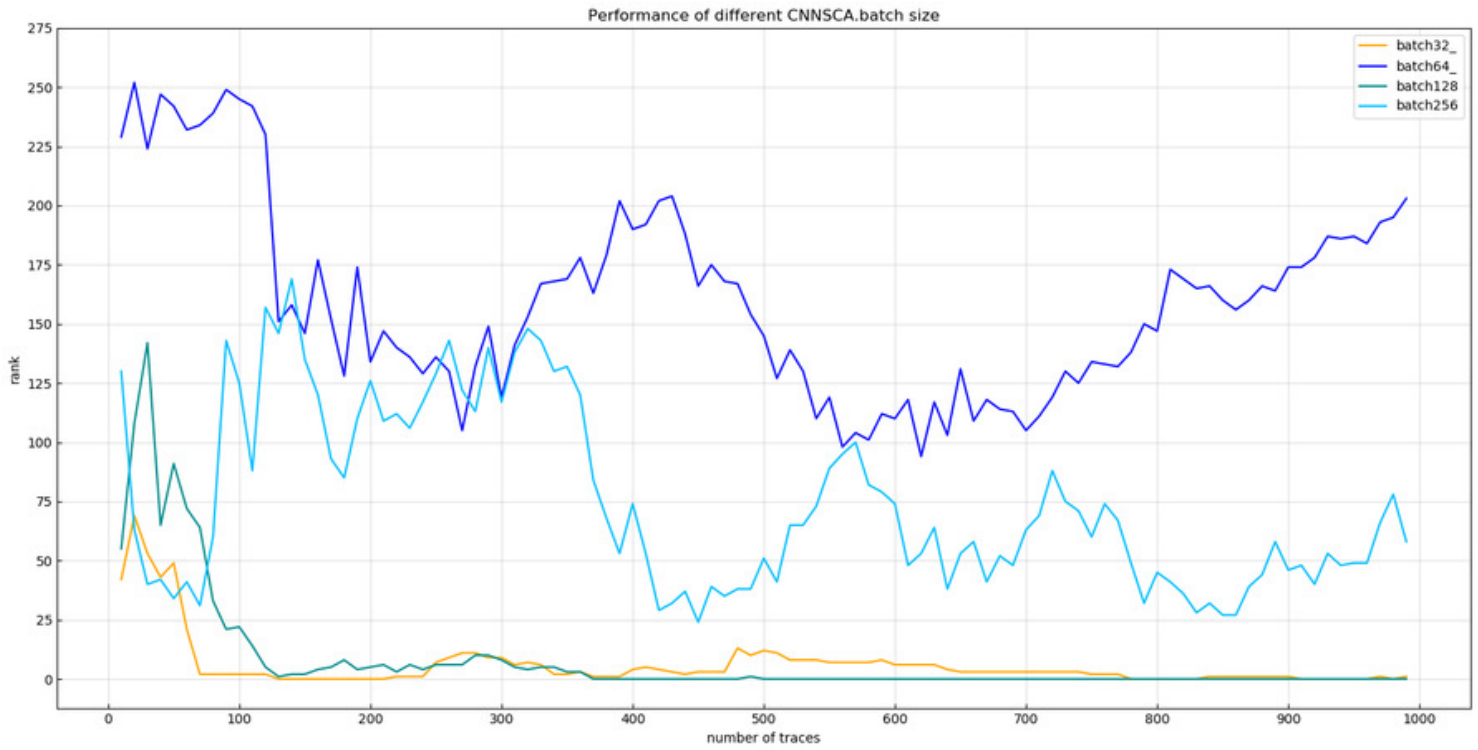


Figure 17

Convergence_of_model_guessing_entropy_under_eight_epochs

Each curve represents the model guessing entropy of eight training epochs. The abscissa represents the number of energy trajectories used in the attack, and the ordinate represents the order of guessing entropy.

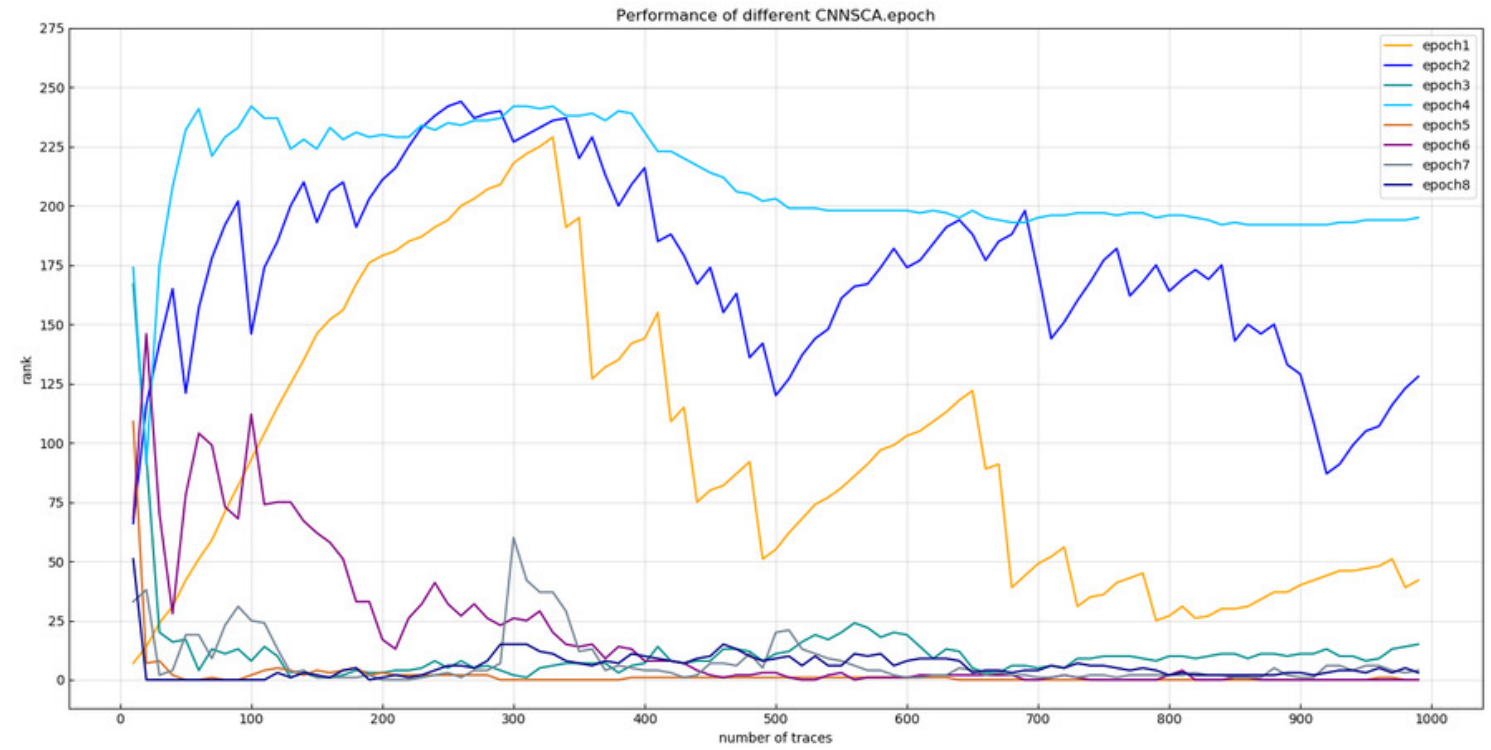




\section{Figure 18}

Convergence_of_model_guessing_entropy_under_four_epochs

Each curve represents the model guessing entropy of four training epochs. The abscissa represents the number of energy trajectories used in the attack, and the ordinate represents the order of guessing entropy.

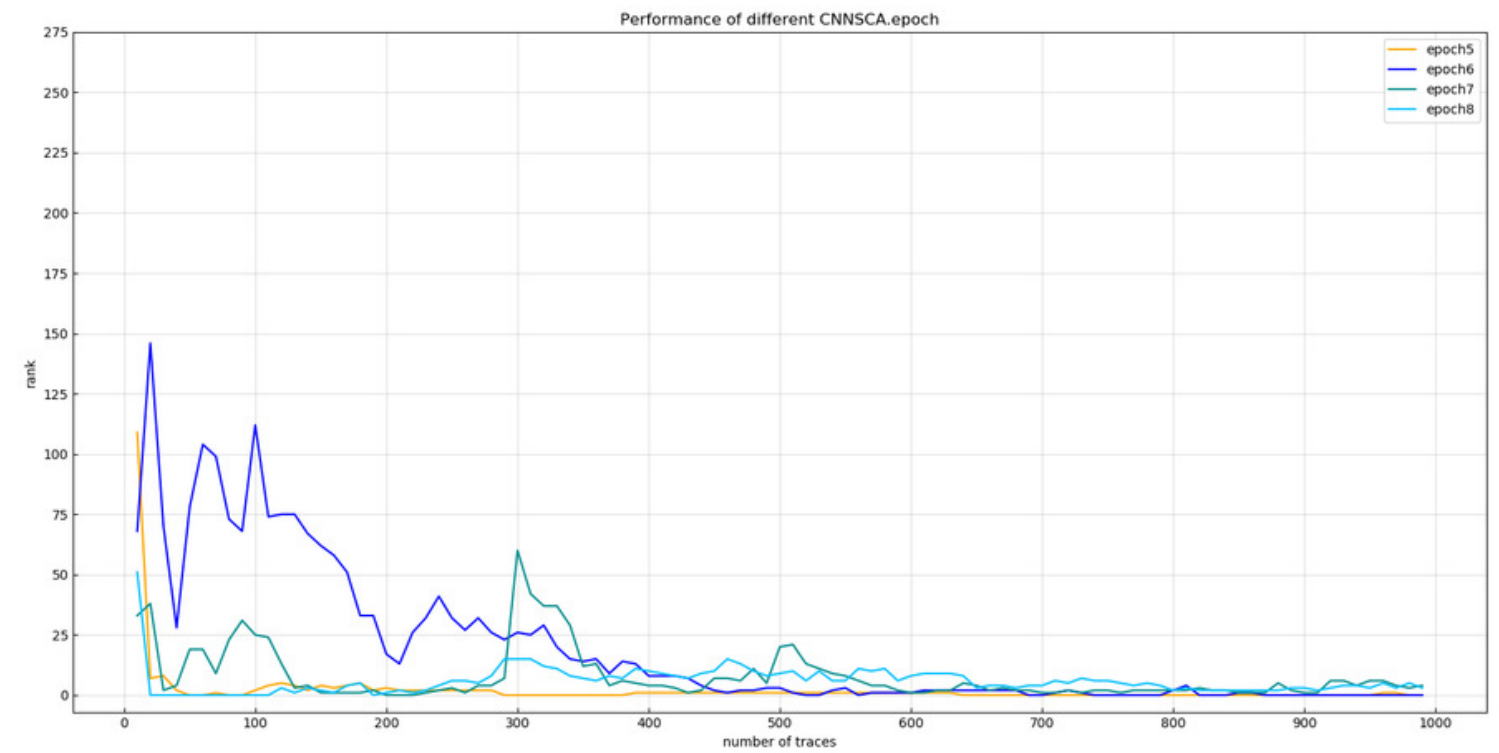




\section{Figure 19}

Convergence_of_model_guessing_entropy_under_five_epochs

Each curve represents the model guessing entropy of five training epochs. The abscissa represents the number of energy trajectories used in the attack, and the ordinate represents the order of guessing entropy.

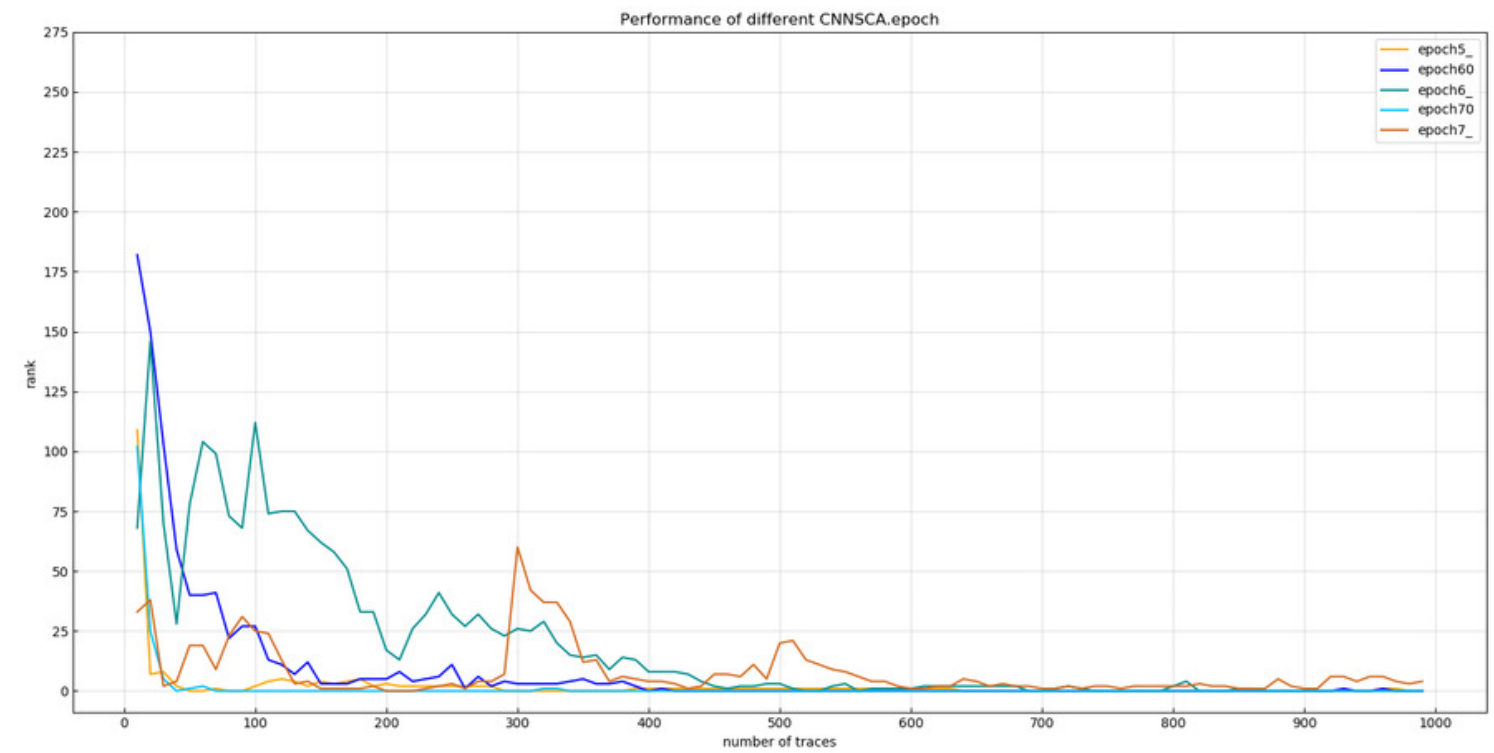


Figure 20

TA,_MLPSCA,_CNNSCAnew_guessing_entropy_convergence

Each curve represents the model guessing entropy of TA, MLPSCA, and CNNSCAnew

respectively. The abscissa represents the number of energy trajectories used in the attack, and the ordinate represents the order of guessing entropy.

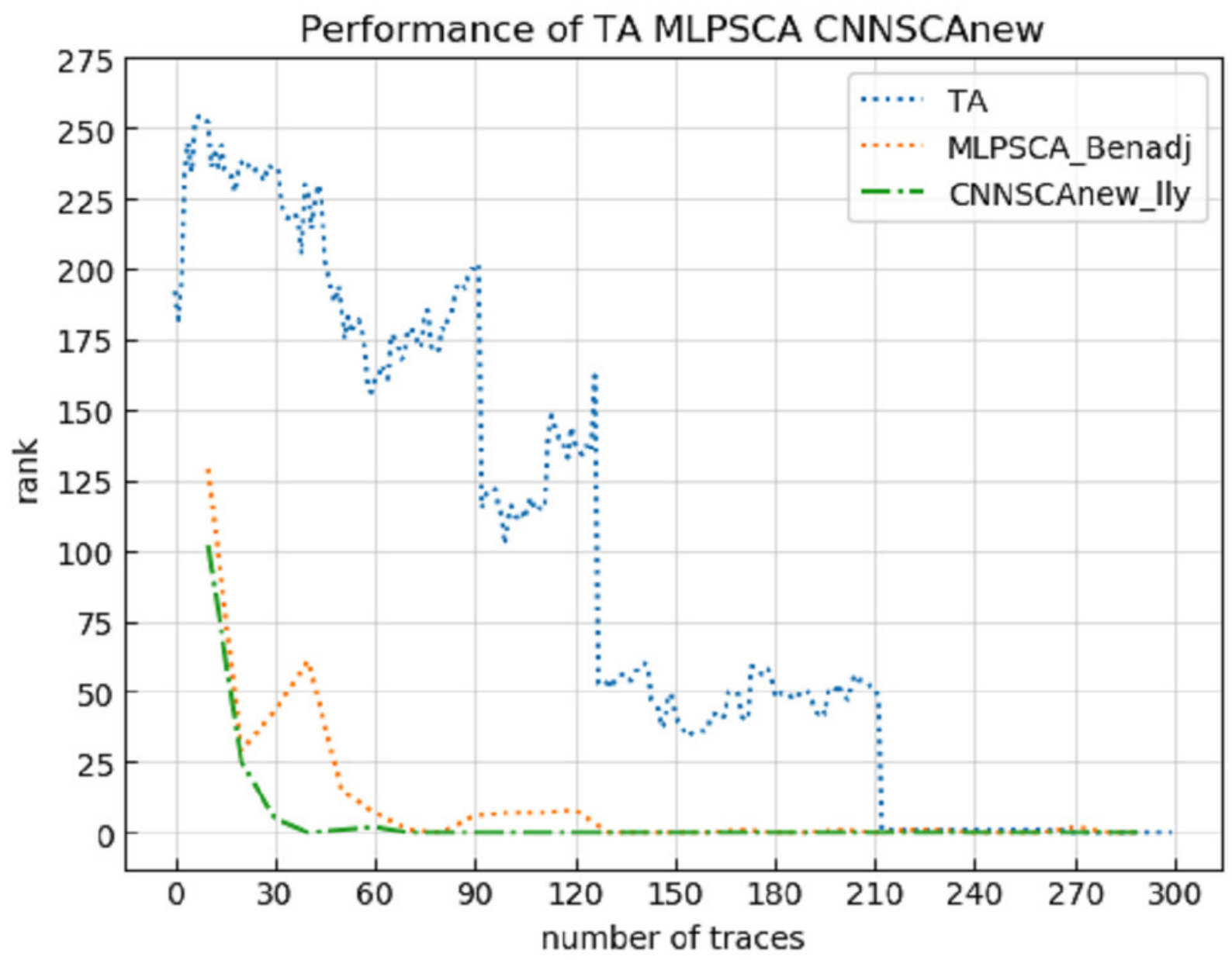




\section{Figure 21}

CNNSCAnew,_VGG-CNNSCA,_Alex-CNNSCA_guessing_entropy_convergence

Each curve represents the model guessing entropy of CNNSCAnew, VGG-CNNSCA and AlexCNNSCA respectively. The abscissa represents the number of energy trajectories used in the attack, and the ordinate represents the order of guessing entropy.

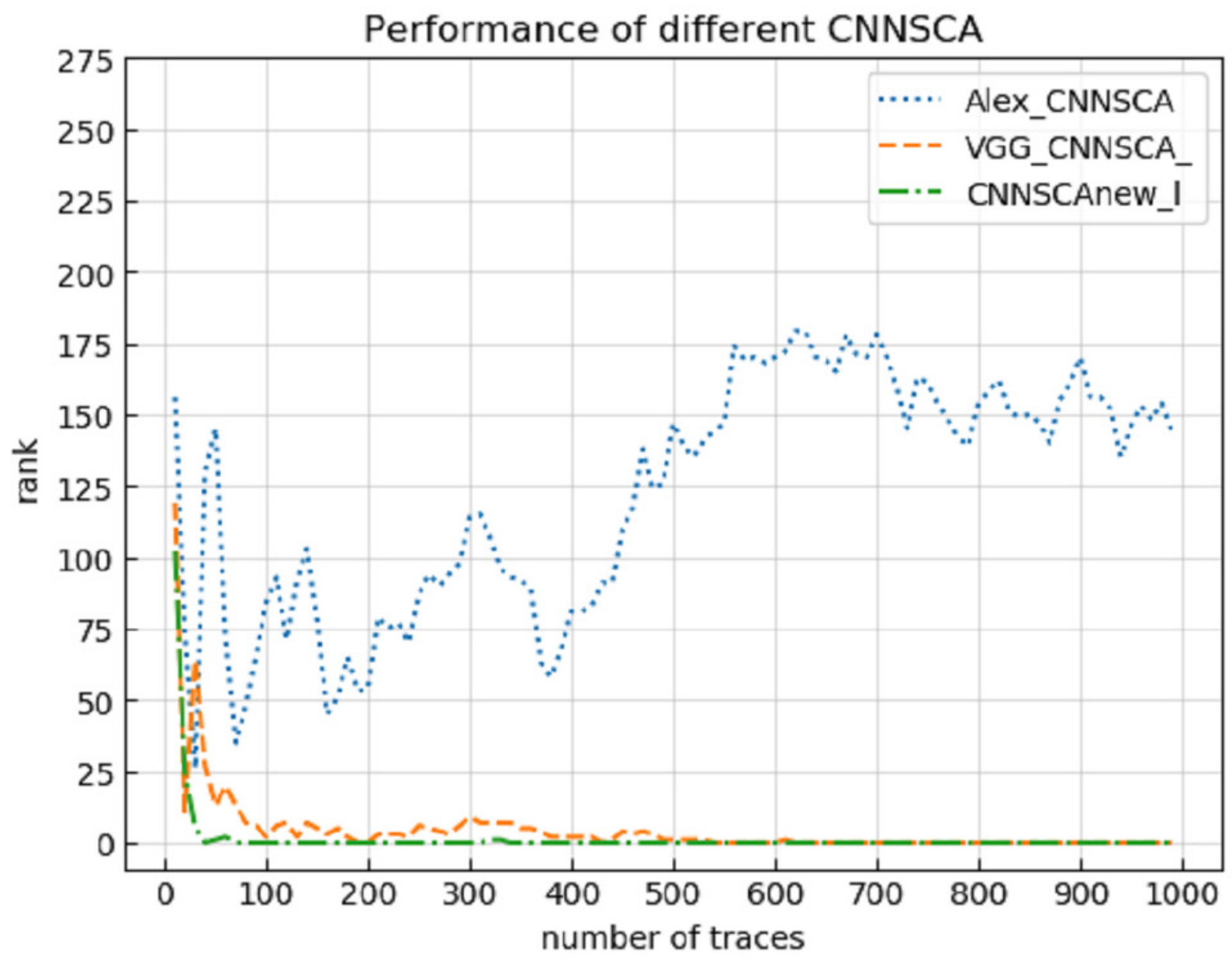




\section{Figure 22}

CNNSCAnew_and_VGG-CNNSCA_training_time

\section{CNNSCAnew}

\section{Running time:1653.920090106 Seconds}

VGG-CNNSCA Running time:2233. 5491708040004 Seconds 


\section{Table $\mathbf{1}$ (on next page)}

CNN with outstanding performance in previous ILSVRC competitions 
Table 1 CNN with outstanding performance in previous ILSVRC competitions

\begin{tabular}{cccccc}
\hline Year & Network / Ranking & val top-1 & val top-5 & test top-5 & Remarks \\
\hline 2012 & Alexnet (Champion) & $36.7 \%$ & $15.4 \%$ & $15.32 \%$ & 7CNNs、 Used data from 2011 \\
2013 & ZFnet (Champion) & -- & -- & $13.51 \%$ & The result on the ZFNet paper is 14.8 \\
2014 & VGG (Runner-up) & $23.7 \%$ & $6.8 \%$ & $6.8 \%$ & Post-race、 2 nets \\
2014 & Googlenet v4 & $16.5 \%$ & $3.1 \%$ & $3.08 \%$ & Post-race、v4+Inception-Res-v2 \\
2015 & (Champion) & -- & -- & $3.57 \%$ & 6 models \\
2016 & Trimpt (Champion) & - & -- & $2.99 \%$ & Public Security III (additional data) \\
2017 & (Champion) & -- & -- & $2.25 \%$ & Momenta and Oxford University \\
\hline
\end{tabular}




\section{Table 2 (on next page)}

CNNSCAbase Configuration 


\begin{tabular}{|c|c|c|c|}
\hline \multicolumn{4}{|c|}{ ConvNet Configuration } \\
\hline \multicolumn{4}{|c|}{ Input(1x700 vector) } \\
\hline Block1 & (Conv3-64)x1 & $\begin{array}{c}\text { Same } \backslash \operatorname{ReL} \\
U\end{array}$ & $\begin{array}{c}\text { AveragePool } \\
(2,2)\end{array}$ \\
\hline Block2 & $\begin{array}{c}(\text { Conv3-128) } \times 1 \\
\text { SE }\end{array}$ & $\begin{array}{c}\text { Same } \backslash R e L \\
U\end{array}$ & $\begin{array}{c}\text { AveragePool } \\
(2,2)\end{array}$ \\
\hline Block3 & $\begin{array}{c}(\text { Conv3-256) } \times 1 \\
\text { SE }\end{array}$ & $\begin{array}{c}\text { Same } \backslash R e L \\
U\end{array}$ & $\begin{array}{c}\text { AveragePool } \\
(2,2)\end{array}$ \\
\hline Block4 & $\begin{array}{c}\text { (Conv3-512) } \times 1 \\
\text { SE }\end{array}$ & $\begin{array}{c}\text { Same } \backslash \text { ReL } \\
U\end{array}$ & $\begin{array}{c}\text { AveragePool } \\
(2,2)\end{array}$ \\
\hline Block5 & $\begin{array}{l}\text { (Conv3- } \\
1024) \times 1\end{array}$ & $\begin{array}{c}\text { Same } \backslash \text { ReL } \\
U\end{array}$ & $\begin{array}{c}\text { AveragePool } \\
(2,2)\end{array}$ \\
\hline \multicolumn{4}{|c|}{$\begin{array}{c}\text { (FC-4096)x2, ReLU } \\
(\text { FC-256)x1, Soft-max }\end{array}$} \\
\hline
\end{tabular}




\section{Table 3 (on next page)}

CNNSCAbase.Conv1-7 Configuration 
Table3 CNNSCAbase.Conv1-7 Configuration

\begin{tabular}{|c|c|c|c|c|c|c|c|}
\hline \multicolumn{8}{|c|}{ Conv Configuration } \\
\hline Block & Conv1 & Conv2 & Conv3 & Conv4 & Conv5 & Conv6 & Conv7 \\
\hline Block1 & $\begin{array}{c}\text { (Conv3- } \\
64) \times 1\end{array}$ & $\begin{array}{c}\text { (Conv3- } \\
64) \times 2\end{array}$ & $\begin{array}{c}\text { (Conv3- } \\
64) \times 2\end{array}$ & $\begin{array}{c}\text { (Conv3- } \\
64) \times 1\end{array}$ & $\begin{array}{c}\text { (Conv3- } \\
64) \times 1\end{array}$ & $\begin{array}{c}\text { (Conv3- } \\
64) \times 1\end{array}$ & $\begin{array}{l}\text { (Conv3- } \\
64) \times 1\end{array}$ \\
\hline \multirow{3}{*}{ Block2 } & (Conv3- & (Conv3- & (Conv3- & (Conv3- & (Conv3- & (Conv3- & (Conv3- \\
\hline & 128)x 1 & 128)x2 & 128)x2 & 128)x2 & 128)x1 & 128)x1 & 128)x1 \\
\hline & $\mathrm{SE}$ & $\mathrm{SE}$ & SE & $\mathrm{SE}$ & $\mathrm{SE}$ & $\mathrm{SE}$ & SE \\
\hline \multirow{3}{*}{ Block3 } & (Conv3- & (Conv3- & (Conv3- & (Conv3- & (Conv3- & (Conv3- & (Conv3- \\
\hline & 256)x1 & 256)x2 & 256)x2 & 256)x2 & 256)x2 & 256)x1 1 & 256)x1 \\
\hline & SE & $\mathrm{SE}$ & $\mathrm{SE}$ & $\mathrm{SE}$ & $\mathrm{SE}$ & SE & SE \\
\hline \multirow{3}{*}{ Block4 } & (Conv3- & (Conv3- & (Conv3- & (Conv3- & (Conv3- & (Conv3- & (Conv3- \\
\hline & $512) \times 1$ & $512) \times 2$ & $512) \times 2$ & $512) \times 2$ & $512) \times 2$ & $512) \times 2$ & $512) \times 1$ \\
\hline & $\mathrm{SE}$ & $\mathrm{SE}$ & SE & $\mathrm{SE}$ & $\mathrm{SE}$ & $\mathrm{SE}$ & SE \\
\hline \multirow{3}{*}{ Block5 } & (Conv3- & (Conv3- & (Conv3- & (Conv3- & (Conv3- & (Conv3- & (Conv3- \\
\hline & 1024)x1 & 1024)x2 & 1024)x3 & 1024)x2 & 1024)x2 & 1024)x2 & 1024)x2 \\
\hline & $\mathrm{SE}$ & $\mathrm{SE}$ & $\mathrm{SE}$ & SE & $\mathrm{SE}$ & $\mathrm{SE}$ & SE \\
\hline
\end{tabular}




\title{
Table 4(on next page)
}

\author{
CNNSCAnew Configuration
}




\begin{tabular}{|c|c|c|c|}
\hline \multicolumn{4}{|c|}{ ConvNet Configuration } \\
\hline \multicolumn{4}{|c|}{ Input(1x700 vector) } \\
\hline Block1 & $($ Conv3-32)x 1 & Same $\backslash$ ReLU & $\begin{array}{c}\text { AveragePool } \\
(2,2)\end{array}$ \\
\hline Block2 & $\begin{array}{l}(\text { Conv3-64)x1 } \\
\text { SEx2 }(1 / 8)\end{array}$ & Same $\backslash$ ReLU & $\begin{array}{l}\text { AveragePool } \\
(2,2)\end{array}$ \\
\hline Block3 & $\begin{array}{l}(\text { Conv3-128)x2 } \\
\text { SEx2 }(1 / 8)\end{array}$ & Same $\backslash R e L U$ & $\begin{array}{l}\text { AveragePool } \\
(2,2)\end{array}$ \\
\hline Block4 & $\begin{array}{l}(\text { Conv3-256)x2 } \\
\text { SEx2 }(1 / 8)\end{array}$ & Same $\backslash$ ReLU & $\begin{array}{l}\text { AveragePool } \\
(2,2)\end{array}$ \\
\hline Block5 & $\begin{array}{l}(\text { Conv3-512)x2 } \\
\text { SEx2 }(1 / 8)\end{array}$ & Same $\backslash$ ReLU & $\begin{array}{c}\text { AveragePool } \\
(2,2)\end{array}$ \\
\hline \multicolumn{4}{|c|}{$\begin{array}{l}\text { (FC-1024)x2, ReLU } \\
(\mathrm{FC}-256) \times 1, \text { Soft-max }\end{array}$} \\
\hline
\end{tabular}




\title{
Table 5 (on next page)
}

\author{
CNNSCAnew Configuration
}


Table5 CNNSCAnew Configuration

\begin{tabular}{|c|c|c|c|}
\hline \multicolumn{4}{|c|}{ ConvNet Configuration } \\
\hline \multicolumn{4}{|c|}{ Input(1x700 vector) } \\
\hline Block1 & (Conv3-32)x1 & $\begin{array}{l}\text { Same } \backslash \text { ReL } \\
U\end{array}$ & $\begin{array}{c}\text { AveragePool } \\
(2,2)\end{array}$ \\
\hline Block2 & $\begin{array}{l}(\text { Conv3-64)x1 } \\
\text { SEx2 }(1 / 8)\end{array}$ & $\begin{array}{c}\text { Same } \backslash \text { ReL } \\
U\end{array}$ & $\begin{array}{l}\text { AveragePool } \\
(2,2)\end{array}$ \\
\hline Block3 & $\begin{array}{c}\text { (Conv3- } \\
128) \times 2 \\
\text { SEx2 }(1 / 8)\end{array}$ & $\begin{array}{c}\text { Same } \backslash \text { ReL } \\
\text { U }\end{array}$ & $\begin{array}{c}\text { AveragePool } \\
(2,2)\end{array}$ \\
\hline Block4 & $\begin{array}{c}\text { (Conv3- } \\
256) \times 2 \\
\text { SEx2 (1/8) }\end{array}$ & $\begin{array}{l}\text { Same } \backslash \text { ReL } \\
\cup\end{array}$ & $\begin{array}{c}\text { AveragePool } \\
(2,2)\end{array}$ \\
\hline Block5 & $\begin{array}{c}\text { (Conv3- } \\
512) \times 2 \\
\text { SEx2 }(1 / 8)\end{array}$ & $\begin{array}{c}\text { Same } \backslash \text { ReL } \\
U\end{array}$ & $\begin{array}{l}\text { AveragePool } \\
(2,2)\end{array}$ \\
\hline \multicolumn{4}{|c|}{$\begin{array}{l}(\text { FC-1024)x2, ReLU } \\
(\text { FC-256) } \times 1 \text {, Soft-max }\end{array}$} \\
\hline \multicolumn{4}{|c|}{$\begin{array}{l}\text { Model compile (crossentropy, RMSprop) } \\
\text { Training parameters }\left(1 \times 10^{-3}, 128,70\right)\end{array}$} \\
\hline
\end{tabular}




\section{Table 6 (on next page)}

Comparative analysis of CNNSCAnew, VGG-CNNSCA and Alex-CNNSCA 
Table 6 Comparative analysis of CNNSCAnew, VGG-CNNSCA and Alex-CNNSCA

\begin{tabular}{|c|c|c|c|c|}
\hline \multicolumn{2}{|c|}{ Three CNNSCA } & CNNSCAnew & VGG-CNNSCA & Alex-CNNSCA \\
\hline \multirow{13}{*}{$\begin{array}{l}\text { CNNSCA } \\
\text { Configuration }\end{array}$} & & $($ Conv3-32)x1 & (Conv11-64)x1 & (Conv11-96)x1 \\
\hline & Convol & Same $\backslash$ ReLU & Same $\backslash R e L U$ & Same $\backslash$ ReLU \\
\hline & & AveragePool $(2,2)$ & AveragePool $(2,2)$ & MaxPool $(2,2)$ \\
\hline & & (Conv3-64)x 1 & (Conv11-128)x1 & $($ Conv5-256)x1 \\
\hline & Convol & SEx2（1/8） & $\begin{array}{l}\text { Same } \backslash \text { ReLU } \\
\text { Selo }\end{array}$ & Same $\backslash$ ReLU \\
\hline & block2 & $\begin{array}{c}\text { Same } \backslash \text { ReLU } \\
\text { AveragePool }(2,2)\end{array}$ & AveragePool $(2,2)$ & MaxPool $(2,2)$ \\
\hline & $\begin{array}{l}\text { Convol } \\
\text { block3 }\end{array}$ & $\begin{array}{c}\text { (Conv3-128)x2 } \\
\text { SEx2 (1/8) } \\
\text { Same \ReLU } \\
\text { AveragePool }(2,2)\end{array}$ & $\begin{array}{c}\text { (Conv11-256)x1 } \\
\text { Same } \backslash \text { ReLU } \\
\text { AveragePool }(2,2)\end{array}$ & $\begin{array}{c}\text { (Conv3-384)x } 1 \\
\text { Same } \backslash \text { ReLU }\end{array}$ \\
\hline & $\begin{array}{l}\text { Convol } \\
\text { block4 }\end{array}$ & $\begin{array}{c}\text { (Conv3-256)x2 } \\
\text { SEx2 }(1 / 8) \\
\text { Same } \backslash \text { ReLU } \\
\text { AveragePool }(2,2)\end{array}$ & $\begin{array}{c}\text { (Conv11-512)x1 } \\
\text { Same } \backslash \text { ReLU } \\
\text { AveragePool }(2,2)\end{array}$ & $\begin{array}{l}(\text { Conv3-384)x1 } \\
\text { Same } \backslash \text { ReLU }\end{array}$ \\
\hline & $\begin{array}{l}\text { Convol } \\
\text { block5 }\end{array}$ & $\begin{array}{c}\text { (Conv3-512)x2 } \\
\text { SEx2 }(1 / 8) \\
\text { Same } \backslash \text { ReLU } \\
\text { AveragePool }(2,2)\end{array}$ & $\begin{array}{c}\text { (Conv11-512)x1 } \\
\text { Same } \backslash \text { ReLU } \\
\text { AveragePool }(2,2)\end{array}$ & $\begin{array}{c}\text { (Conv3-256)x1 } \\
\text { Same } \backslash \text { ReLU } \\
\text { MaxPool }(3,3)\end{array}$ \\
\hline & dense layer & $\begin{array}{l}(\mathrm{FC}-1024) \times 2, \text { ReLU } \\
(\mathrm{FC}-256) \times 1, \text { Soft-max }\end{array}$ & $\begin{array}{l}(\mathrm{FC}-4096) \times 2, \text { ReLU } \\
(\mathrm{FC}-256) \times 1, \text { Soft-max }\end{array}$ & $\begin{array}{l}(\mathrm{FC}-4096) \times 2, \text { ReLU } \\
(\mathrm{FC}-256) \times 1, \text { Soft-max }\end{array}$ \\
\hline & $\begin{array}{l}\text { Learning } \\
\text { rate }\end{array}$ & $1 \times 10^{-3}$ & $10^{-5}$ & $10^{-2}$ \\
\hline & Batch size & 128 & 200 & 10 \\
\hline & epoch & 70 & 75 & 20 \\
\hline \multirow{2}{*}{$\begin{array}{l}\text { CNNSCA } \\
\text { Performance }\end{array}$} & $\begin{array}{l}\text { calculating } \\
\text { time }\end{array}$ & 28 minute & 37 minute & 2 hour \\
\hline & $\begin{array}{l}\text { Guess } \\
\text { entropy }\end{array}$ & 61 & 650 & Did not converge \\
\hline
\end{tabular}

